\title{
Theoretical Investigation of some Antimalarial Sulfonamides as COVID-19 Drug Utilizing Computational Calculations and Molecular Docking Study
}

\author{
Asmaa M Fahim $^{1, * \mathbb{D}}$, Eman H. I. Ismael ${ }^{2}$ (D) \\ 1 Green Chemistry Department, National Research Center, Dokki, P.O. Box.12311, Cairo, Egypt \\ 2 Organometallic and Organmetalloid Chemistry Department, National Research Centre, Dokki, P.O. Box.12311, Cairo, \\ Egypt \\ * Correspondence: asmaamahmoud8521@gmail.com;
}

Scopus Author ID 56765412200

Received: 22.02.2021; Revised: 4.04.2021; Accepted: 8.04.2021; Published: 26.04.2021

\begin{abstract}
Reactivity investigation of $\mathrm{N}$-(phenylsulfonyl)acetamide derivatives with nitrogen nucleophiles to give the corresponding aminothiazole, aminooxazole, $2 \mathrm{H}$-benzo[b][1,4]oxazin-3-yl, 2H-benzo[b][1,4]thiazin-3-yl, quinoxalin-2-yl, pyridin-4-ylamino, benzo[d]thiazol-2-ylthio and 1Hbenzo[d]imidazol-2-ylthio derivatives; respectively. The sulphonamides were examined in vitro antimalarial activity and characterized their ADMET properties, with $\mathrm{IC}_{50}$ values of $<30 \mu \mathrm{M}$. The synthesized sulfonamide had c-LogP values between 2 and (mean value 2.23) and MW(molecular weight) less than 400, and the common and didn't exhibition cytotoxicity at the concentration confirmed. Sulphonamide 6 a was equipped the excellent antimalarial activity through $\mathrm{IC}_{50}=1.2 \mu \mathrm{M}$ with had the best selectivity index (9.0) due to the occurrence of quinoxaline moiety attached to the sulfonamide ring system. Additionally, the theoretical calculations of most active compounds $4 \mathrm{a}, 5 \mathrm{c}$, and 6a exhibited that value of $\Delta \mathrm{E}$ so small which growth tendency of electron transfer from HOMOLUMO energy gap due to existence of benzo[b][1,4]oxazin-3-yl,benzo[b][1,4]thiazin-3-yl and quinoxalin-2-yl rings attached to sulfonamide ring. Additionally, The molecular docking study of these synthesized compounds revealed small energy affinity against Plasmepsin-1 (PDBID:1j3i) and Plasmepsin-2 (PDBID:1n81) and shorter bond length. Also, the most active compounds 4a, 5c, and 6a were docked on main protease (SARS-CoV-2) (PDBID: 6lu7) and Spike Glycoprotein (SARS-CoV-2) (PDBID:6vxx) to calculate the binding energy of these fused heterocycles sulfonamides.
\end{abstract}

Keywords: fused sulfonamide; antimalarial activity; computational calculations; molecular docking; COVID-19.

(C) 2021 by the authors. This article is an open-access article distributed under the terms and conditions of the Creative Commons Attribution (CC BY) license (https://creativecommons.org/licenses/by/4.0/).

\section{Introduction}

The COVID-19, which was initiated through SARS-CoV-2, occurred as contamination and rapidly degree to all countries and is mainly extent through interaction with infected dewdrops of saliva from the nose when diseased people cough sneeze. The first infection of coronavirus in the world was in 1960 [1, 2].

Coronavirus is a domestic of stranded- positive intelligence of RNA virus. Additionally, it was alienated into four genera: $\alpha, \beta, \gamma$, and $\delta$. Coronaviruses of $\alpha$ and $\beta$ genera typically contaminate mammals and humans while the type of $\gamma$ and $\delta$ genera mainly infect birds. That explanation is affording to the phylogenetic analysis and genome structure of coronaviruses [3-5]. 
Besides the sulfonamide moieties have an extensive spectrum of biological actions, for example, a monoamine oxidase inhibitor, anticonvulsant, antibacterial, hypotensive, antipyretic, and anti-inflammatory, antimicrobial and anthelmintic activities [6-13]. Likewise, the target nucleus indicates the core unit in a variety of drugs, such as Sulfafurazole (I), Chlorpropamide (II), Ethoxzolamide (III), and Sulfamethoxypyridazine (IV) and Sulfadoxine (V) as displayed in (Figure 1).

The malaria parasites grow rapidly in the liver and then in the red blood cells of the infected person [14]. In addition, Sulfadoxine (V) is an ultra-long-lasting sulfonamide earlier utilized in alignment with pyrimethamine to delicacy or avoid malaria [15, 16]. Due to high resistance levels, its occupation is no longer recommended routinely [17-22]. The antimalarial achievement of sulfadoxine standbys to be related to home metabolism of the causative agent, Plasmodium falciparum [23-25].

Nevertheless, the extent of sulfadoxine-resistant P.Falciparum strains has dashed of global malaria eradication endemic areas. Consequently, many purposes are dedicated to designing and synthesizing innovative and mechanically varied compounds with potential antimalarial activity [26-28].

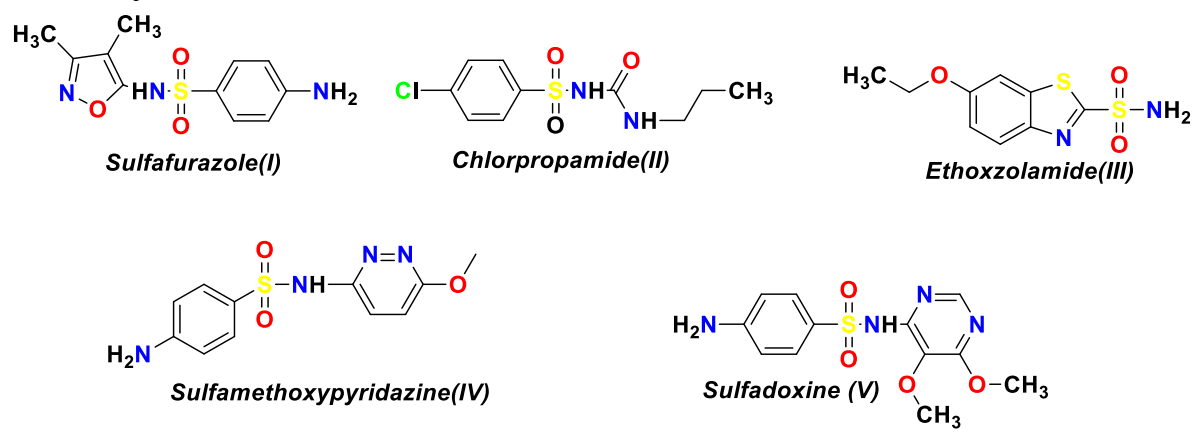

Figure 1. Some drugs of sulfonamide.

This investigation synthesized the innovative sulfonamide via reaction of active $\mathrm{N}$ (phenylsulfonyl)acetamide derivatives with nitrogen compounds and assessing their antimalarial activities. The computational study of most active antimalarial compounds $4 \mathrm{a}, 5 \mathrm{c}$, and 6c utilizes DFT B3LYP/6-31G(d) level. Moreover, the molecular docking study of these compounds against Plasmepsin-1 (PDBID:1j3i) and Plasmepsin-2 (PDBID:1n81)and also with (SARS-CoV-2 main protease) (PDBID: 6lu7) and (SARS-CoV-2 Spike Glycoprotein) (PDBID:6vxx) to relate for additional biological evaluation.

\section{Materials and Methods}

\subsection{General procedure.}

Melting points were examined on a Gallenkamp apparatus. -FT-IR spectra were designated on (Shimadzu FT-IR $8101 \mathrm{PC}$ infrared spectrophotometer). The ${ }^{1} \mathrm{H}-\mathrm{NMR}$ spectrum was resolute in DMSO at $300 \mathrm{MHz}$ on a (Varian Mercury VX 300 NMR spectrometer) and ${ }^{13} \mathrm{C}$ at $75 \mathrm{MHz}$ ) utilizing TMS as an internal standard. The mass spectrum was recorded on a (Shimadzu GCMS-QP 1000 EX mass spectrometer at $70 \mathrm{eV}$ ). Elemental analyses were determined via at the Microanalytical Center /Cairo University/Giza, Egypt. 


\subsubsection{Materials and reagents.}

Sulfonyl chloride derivatives, 2-bromoacetamide, thiourea, urea, ammonium hydroxide, 2-aminophenol, 2-aminobenzothiazole, o-phenylenediamine, 4-aminopyridine, benzo $[d]$ thiazole-2-thiol, and $1 H$ benzo $[d]$ imidazole-2-thiol were purchased from Aldrich Chemical CO. Solvents were purchased from Aldrich Chemical CO.

2.1.2. Reaction of 2-Bromo-N-(phenylsulfonyl)acetamide derivatives(1a-c) with urea derivatives.

Ethanolic solution of the 2-bromo- $N$-(phenylsulfonyl)acetamide derivative 1a-c (3.88 $\mathrm{g}, 10 \mathrm{mmol}$ ) with the appropriate urea derivatives was refluxed for 4 hours treated with ammonium hydroxide solution until $\mathrm{pH}=9$. The solid was formed was filtered off and washed with water, dried, and finally crystallized from $(\mathrm{DMF} / \mathrm{EtOH})$ and $\left(\mathrm{EtOH} / \mathrm{H}_{2} \mathrm{O}\right)$, respectively [8].

$\mathrm{N}$-(2-aminothiazol-4-yl)-4-chlorobenzenesulfonamide(2a): Brown powder, in 72\% yield, m.p. $=185-187^{\circ} \mathrm{C}, \mathrm{C}_{9} \mathrm{H}_{8} \mathrm{ClN}_{3} \mathrm{O}_{2} \mathrm{~S}_{2}$ (288.97), Analysis\% Calcd (Found): C: $37.31(37.32$ ), $\mathrm{H}: 2.78(2.80), \mathrm{Cl}: 12.24(12.26), \mathrm{N}: 14.50(14.53), \mathrm{S}: 22.13$ (22.15), IR (KBr) ${ }_{\max } / \mathrm{cm}^{-1}: 3350$ $(\mathrm{NH}), 3010\left(\mathrm{NH}_{2}\right), 1302(\mathrm{~S}=\mathrm{O}) .{ }^{1} \mathrm{H}$ NMR (DMSO-d $)$ ): $\delta$ 6.60(s, 1H, HC, thiazole ring), 7.25 (s, $2 \mathrm{H}, \mathrm{H}_{2} \mathrm{~N}-\mathrm{D}_{2} \mathrm{O}$ exchangeable), $7.69(\mathrm{~d}, 2 \mathrm{H}, \mathrm{HC}$ aromatic, $\mathrm{J}=7.5 \mathrm{~Hz}), 8.02(\mathrm{~d}, 2 \mathrm{H}, \mathrm{HC}$ aromatic, $\mathrm{J}=3.2 \mathrm{~Hz}), 10.23\left(\mathrm{~s}, 1 \mathrm{H}, \mathrm{HN}-\mathrm{D}_{2} \mathrm{O}\right.$ exchangeable), ${ }^{13} \mathrm{C}$ NMR (DMSO-d6): $\delta 112(\mathrm{CH}), 128.2(\mathrm{CH})$, 137.8(CH), 140(CH), 169(CH), MS (m/z, r.i.\%): 288 ( $\left.\mathrm{M}^{+}, 100 \%\right), 290\left(\mathrm{M}^{+2}, 32 \%\right), 95(23 \%)$.

$\mathrm{N}$-(2-aminothiazol-4-yl)-4-methylbenzenesulfonamide (2b): Yellow solid, in 72\% yield,m.p.=134- $136^{\circ} \mathrm{C}, \mathrm{C}_{10} \mathrm{H}_{11} \mathrm{~N}_{3} \mathrm{O}_{2} \mathrm{~S}_{2}$ (269.34), Analysis\% Calcd (Found): C: 44.59(44.60), H: 4.12(4.19), N: 15.60(15.63), S: 23.79(23.49). IR (KBr) $\max / \mathrm{cm}^{-1}: 3215(\mathrm{NH}), 3100-3025\left(\mathrm{NH}_{2}\right)$, $1289(\mathrm{~S}=\mathrm{O}) .{ }^{1} \mathrm{H}$ NMR (DMSO-d $): \delta 2.43\left(\mathrm{~s}, 3 \mathrm{H}, \mathrm{H}_{3} \mathrm{C}\right), 4.65\left(\mathrm{~s}, 2 \mathrm{H}, \mathrm{H}_{2} \mathrm{~N}-\mathrm{D}_{2} \mathrm{O}\right.$ exchangeable), 6.56(s, 1H, HC, thiazole), 7.25(d, 2H, HC aromatic, $\mathrm{J}=3.2 \mathrm{~Hz}), 7.73(\mathrm{~d}, 2 \mathrm{H}, \mathrm{HC}$ aromatic, $\mathrm{J}=8.5 \mathrm{~Hz}), 10.29\left(\mathrm{~s}, 1 \mathrm{H}, \mathrm{HN} \mathrm{D}_{2} \mathrm{O}\right.$ exchangeable). ${ }^{13} \mathrm{C}$ NMR (DMSO-d $): \delta 19.2\left(\mathrm{CH}_{3}\right), 112(\mathrm{CH})$, 128.2(CH), 129.3(CH), 137.8(CH), 140(CH), 169(CH), MS (m/z, r.i.\%): 269( $\left.\mathrm{M}^{+}, 88 \%\right)$, $170(55 \%), 114(29 \%)$.

N-(2-aminothiazol-4-yl)benzenesulfonamide (2c): Reddish brown, in 70\% yield, $\mathrm{C}_{9} \mathrm{H}_{9} \mathrm{~N}_{3} \mathrm{O}_{2} \mathrm{~S}_{2}$ (255.32), m.p.=136- $138^{\circ} \mathrm{C}$, Analysis\% Calcd (Found): C: 42.34(42.40), H: 3.55(3.58), N: 16.46(16.49), S: $25.11(25.15)$. IR (KBr) $\max / \mathrm{cm}^{-1}: 3215(\mathrm{NH}), 3100-3025\left(\mathrm{NH}_{2}\right)$, $1289(\mathrm{~S}=\mathrm{O}) .{ }^{1} \mathrm{H}$ NMR (DMSO-d $): \delta 6.58\left(\mathrm{~s}, 1 \mathrm{H}, \mathrm{HC}\right.$ thiazole proton), $7.17\left(\mathrm{~s}, 2 \mathrm{H}, \mathrm{H}_{2} \mathrm{~N}_{-} \mathrm{D}_{2} \mathrm{O}\right.$ exchangeable), 7.78-7.82(m, 5H, HArs), 10.33(s, 1H, HN - $\mathrm{D}_{2} \mathrm{O}$ exchangeable). ${ }^{13} \mathrm{C}$ NMR (DMSO-d6): $\delta 110(\mathrm{CH}), 129.3(\mathrm{CH}), 131(\mathrm{CH}), 137(\mathrm{CH}), 140(\mathrm{CH}), 170(\mathrm{CH}), \mathrm{MS}(\mathrm{m} / \mathrm{z}, \mathrm{r} . \mathrm{i} . \%)$ : $255\left(\mathrm{M}^{+}, 100 \%\right), 156(10 \%), 114(25 \%)$.

$\mathrm{N}$-(2-aminooxazol-4-yl)-4-chlorobenzenesulfonamide (3a): Off white powder, in 62\% yield, m.p.=166- $168^{\circ} \mathrm{C}, \mathrm{C}_{9} \mathrm{H}_{8} \mathrm{ClN}_{3} \mathrm{O}_{3} \mathrm{~S},(273.70)$, Analysis\% Calcd (Found): C: $39.50(39.53)$, $\mathrm{H}: 2.95(2.98), \mathrm{Cl}: 12.95(12.93), \mathrm{N}: 15.35(15.40), \mathrm{S}: 11.71(11.76)$. IR (KBr) $\mathrm{max} / \mathrm{cm}^{-1}$ : 3403(NH), 3230-3125( $\left.\mathrm{NH}_{2}\right), 1310(\mathrm{~S}=\mathrm{O}) .{ }^{1} \mathrm{H}$ NMR (DMSO-d6): $\delta 6.23\left(\mathrm{~s}, 2 \mathrm{H}, \mathrm{H}_{2} \mathrm{~N} \mathrm{D} \mathrm{D}_{2} \mathrm{O}\right.$ exchangeable), 7.12(s, 1H, HC oxazole), $7.69(\mathrm{~d}, 2 \mathrm{H}, \mathrm{HC}$ aromatic, $\mathrm{J}=1.5 \mathrm{~Hz}), 8.02(\mathrm{~d}, 2 \mathrm{H}, \mathrm{HC}$ aromatic, $\mathrm{J}=7.5 \mathrm{~Hz}), 10.25\left(\mathrm{~s}, 1 \mathrm{H}, \mathrm{HN} \mathrm{D} 2 \mathrm{O}\right.$ exchangeable). ${ }^{13} \mathrm{C}$ NMR (DMSO-d $): \delta 126(\mathrm{CH})$, 128(CH), 137(CH), 165(CH), MS (m/z, r.i.\%): 273( $\left.\mathrm{M}^{+}, 100 \%\right), 275\left(\mathrm{M}^{+2}, 33 \%\right), 189(32 \%)$, $98(25 \%)$.

$\mathrm{N}$-(2-aminooxazol-4-yl)-4-methylbenzenesulfonamide (3b): White solid in $64 \%$ yield, m.p. $=141-143^{\circ} \mathrm{C}, \mathrm{C}_{10} \mathrm{H}_{11} \mathrm{~N}_{3} \mathrm{O}_{3} \mathrm{~S}$ (253.28), Analysis\% Calcd (Found): C: 47.42(47.40), H: 
4.38(4.40), N: 16.59(16.57), S:12.66(12.69), IR (KBr) $\max / \mathrm{cm}^{-1}: 3403(\mathrm{NH}), 3230-3125\left(\mathrm{NH}_{2}\right)$, $1310(\mathrm{~S}=\mathrm{O}) .{ }^{1} \mathrm{H}$ NMR (DMSO-d6): $\delta 2.42\left(\mathrm{~s}, 3 \mathrm{H}, \mathrm{H}_{3} \mathrm{C}\right), 6.50\left(\mathrm{~s}, 2 \mathrm{H}, \mathrm{H}_{2} \mathrm{~N} \mathrm{D}_{2} \mathrm{O}\right.$ exchangeable), 7.12(s, 1H, HC oxazole), 7.63(d, 2H, HC aromatic, $\mathrm{J}=3.2 \mathrm{~Hz}), 8.02(\mathrm{~d}, 2 \mathrm{H}, \mathrm{HC}$ aromatic, $\mathrm{J}=$ 7.5Hz), 10.29(s, 1H, HN- D $2 \mathrm{O}$ exchangeable). ${ }^{13} \mathrm{C}$ NMR (DMSO-d6): $\delta 19.2\left(\mathrm{CH}_{3}\right), 125.4(\mathrm{CH})$,

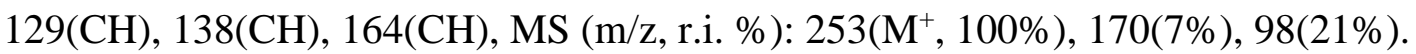

$\mathrm{N}$-(2-aminooxazol-4-yl)benzenesulfonamide (3c): Brown solid, in 64\% yield, m.p. $=133-135^{\circ} \mathrm{C} \mathrm{C}_{9} \mathrm{H}_{9} \mathrm{~N}_{3} \mathrm{O}_{3} \mathrm{~S}$ (239.25), Analysis\% Calcd (Found): C: 45.18(45.20), H: 3.79(3.80), N: 17.56(17.55), S: 13.40 (13.42), IR ( KBr) max $/ \mathrm{cm}^{-1}: 3403(\mathrm{NH}), 3230-3125\left(\mathrm{NH}_{2}\right)$, $1310(\mathrm{~S}=\mathrm{O}) .{ }^{1} \mathrm{HNMR}$ (DMSO-d $): \delta 6.53\left(\mathrm{~s}, 2 \mathrm{H}, \mathrm{H}_{2} \mathrm{~N} \mathrm{D}_{2} \mathrm{O}\right.$ exchangeable), 7.10(s, 1H, HC oxazole), 7.75-7.83(m, 5H, HArs), 10.29(s, 1H, HN- $\mathrm{D}_{2} \mathrm{O}$ exchangeable). ${ }^{13} \mathrm{C}$ NMR (DMSOd6): $\delta 125.4(\mathrm{CH}), 127(\mathrm{CH}), 129(\mathrm{CH}), 131(\mathrm{CH}), 138(\mathrm{CH}), 163(\mathrm{CH}), \mathrm{MS}(\mathrm{m} / \mathrm{z}, \mathrm{r} . \mathrm{i} . \%): 239\left(\mathrm{M}^{+}\right.$, $100 \%), 156(12 \%), 98(25 \%)$.

2.1.3. Reaction of 2-bromo-N-(phenylsulfonyl)acetamidederivatives(1a-c) with amino heterocyclic derivatives.

2-Bromo-N-(phenylsulfonyl) acetamide derivative (1a-c) $(0.33 \mathrm{~g}, 2 \mathrm{mmol})$ reacts the aromatic amine derivative (2 mmol) [8] in ethanol (10 ml), and $\mathrm{H}_{2} \mathrm{SO}_{4}(5 \mathrm{ml})$ were refluxed for 6-8 hours. The reaction mixture was evaporated in vacuo, and the residual solid was collected by filtration, washed with ethanol, dried, and finally recrystallized from the suitable solvent to afford the corresponding heterocyclic derivatives 4a-c, 5a-c and 6a-c [8] recrystallized from (EtOH/DMF).

$\mathrm{N}$-(2H-Benzo[b][1,4]oxazin-3-yl)-4-chlorobenzenesulfonamide(4a):Yellow solid in $62 \%$ yield, m.p. $=192-193^{\circ} \mathrm{C} \quad \mathrm{C}_{14} \mathrm{H}_{11} \mathrm{ClN}_{2} \mathrm{O}_{3} \mathrm{~S}(322.76)$, Analysis\% Calcd (Found): C: 52.10(52.08), H: 3.44(3.42), Cl: 10.98(10.96), N: 8.68(8.64), S: 9.93 (9.90), IR (KBr) max/cm ${ }^{1}: 3325(\mathrm{NH}), 1259(\mathrm{~S}=\mathrm{O}) .{ }^{1} \mathrm{H}$ NMR (DMSO-d $): \delta 4.51(\mathrm{~s}, 2 \mathrm{H}, \mathrm{HC}), 7.28(\mathrm{dd}, 2 \mathrm{H}, \mathrm{HC}$ aromatic, $\mathrm{J}=12 \mathrm{~Hz}), 7.55(\mathrm{t}, 1 \mathrm{H}, \mathrm{HC}$ aromatic, $\mathrm{J}=1.2 \mathrm{~Hz}), 7.60\left(\mathrm{~s}, 2 \mathrm{H}, \mathrm{H}_{2} \mathrm{C}\right.$ aromatic, $\left.\mathrm{J}=7.5 \mathrm{~Hz}\right), 7.69(\mathrm{~d}$, $2 \mathrm{H}, \mathrm{HC}$ aromatic, $\mathrm{J}=7.5 \mathrm{~Hz}), 7.74\left(\mathrm{~s}, 1 \mathrm{H}, \mathrm{HN} \mathrm{D}_{2} \mathrm{O}\right.$ exchangeable $), 8.20(\mathrm{~s}, 1 \mathrm{H}, \mathrm{HC}=\mathrm{N}$ aromatic), ${ }^{13} \mathrm{C}$ NMR (DMSO-d $): \delta 107.2(\mathrm{CH}), 116(\mathrm{CH}) 121(\mathrm{CH}), 125.1(\mathrm{CH}), 128.2(\mathrm{CH})$, 162.3(CH), MS (m/z, r.i.\%): 322( $\left.\mathrm{M}^{+}, 100 \%\right), 189(7 \%), 147(45 \%)$.

N-(2H-Benzo[b][1,4]oxazin-3-yl)-4-methylbenzenesulfonamide(4b): Dark brown 67\% yield, m.p. $=191-192^{\circ} \mathrm{C}, \mathrm{C}_{15} \mathrm{H}_{14} \mathrm{~N}_{2} \mathrm{O}_{3} \mathrm{~S}(302.35)$, Analysis\% Calcd (Found): C: $59.59(59.60), \mathrm{H}$ : 4.67(4.65), N: 9.27(9.30), S: 10.60(10.63). IR (KBr) $\max / \mathrm{cm}^{-1}: 3324(\mathrm{NH}), 1228(\mathrm{~S}=\mathrm{O}) .{ }^{1} \mathrm{H}$ NMR (DMSO-d $): \delta 2.42\left(\mathrm{~s}, 3 \mathrm{H}, \mathrm{H}_{3} \mathrm{C}\right), 4.44(\mathrm{~s}, 2 \mathrm{H}, \mathrm{HC}), 7.26(\mathrm{dd}, 2 \mathrm{H}, \mathrm{HC}$ aromatic, J=12Hz), 7.43(t, $1 \mathrm{H}, \mathrm{HC}$ aromatic, $\mathrm{J}=1.2 \mathrm{~Hz}), 7.59\left(\mathrm{~s}, 2 \mathrm{H}, \mathrm{H}_{2} \mathrm{C}\right.$ aromatic, $\left.\mathrm{J}=7.5 \mathrm{~Hz}\right), 7.70(\mathrm{~d}, 2 \mathrm{H}, \mathrm{HC}$ aromatic, $\mathrm{J}=7.5 \mathrm{~Hz}), 7.74\left(\mathrm{~s}, 1 \mathrm{H}, \mathrm{HN} \mathrm{D} \mathrm{O}_{2} \mathrm{O}\right.$ exchangeable), 8.15(s,1H, $\mathrm{HC}=\mathrm{N}$ aromatic),${ }^{13} \mathrm{C}$ NMR (DMSOd6): $\delta 19.2\left(\mathrm{CH}_{3}\right)$ 107.2(CH), 116.2(CH) 121(CH), 125.1(CH), 128.2(CH), 162.3(CH), MS (m/z, r.i.\%): 302( $\left.\mathrm{M}^{+}, 100 \%\right), 170(7 \%), 147(33 \%)$.

$\mathrm{N}-(2 \mathrm{H}-$ Benzo[b][1,4]oxazin-3-yl)benzenesulfonamide(4c): Brown solid, $67 \%$ yield, m.p. $=188-190^{\circ} \mathrm{C}, \quad \mathrm{C}_{14} \mathrm{H}_{12} \mathrm{~N}_{2} \mathrm{O}_{3} \mathrm{~S} \quad$ (288.32), Analysis\% Calcd (Found): C:58.32(58.30),H:4.20(4.18), N: 9.72(9.65), S: 11.12 (11.09). IR (KBr) $\mathrm{max} / \mathrm{cm}^{-1}: 3320(\mathrm{NH})$, $1303(\mathrm{~S}=\mathrm{O}) .{ }^{1} \mathrm{H}$ NMR (DMSO-d6): $\delta$ 4.44(s, 2H, HC), 7.26(dd, $2 \mathrm{H}, \mathrm{HC}$ aromatic, J=12Hz), 7.43(t, $1 \mathrm{H}, \mathrm{HC}$ aromatic, $\mathrm{J}=1.2 \mathrm{~Hz}), 7.55-7.68(\mathrm{~m}, 5 \mathrm{H}, \mathrm{ArsH}), 7.70\left(\mathrm{~s}, 1 \mathrm{H}, \mathrm{HN} \mathrm{D}_{2} \mathrm{O}\right.$ exchangeable), 8.15(s,1H, $\mathrm{HC}=\mathrm{N}$ aromatic), $\mathrm{MS}(\mathrm{m} / \mathrm{z}, \mathrm{r.i} . \%): 288\left(\mathrm{M}^{+}, 100 \%\right), 156(12 \%)$, $147(32 \%)$.

N-(2H-Benzo[b][1,4]thiazin-3-yl)-4-chlorobenzenesulfonamide(5a): Reddish yellow , $66 \%$ yield, m.p. $=204-206^{\circ} \mathrm{C}, \mathrm{C}_{14} \mathrm{H}_{11} \mathrm{ClN}_{2} \mathrm{O}_{2} \mathrm{~S}_{2}$, (338.83), Analysis\% Calcd (Found): C: 
49.63(49.60), H:3.27(3.32), Cl: 10.46 (10.49), N: 8.27(8.30), S: 18.92 (18.90).IR (KBr) $\mathrm{max} / \mathrm{cm}^{-}$ 1: 3301(NH), $1255(\mathrm{~S}=\mathrm{O}) .{ }^{1} \mathrm{H}$ NMR (DMSO-d6): $84.33(\mathrm{~s}, 2 \mathrm{H}, \mathrm{HC}), 7.20-7.22(\mathrm{dd}, 2 \mathrm{H}, \mathrm{HC}$ aromatic, $\mathrm{J}=12 \mathrm{~Hz}), 7.42(\mathrm{t}, 1 \mathrm{H}, \mathrm{HC}$ aromatic, $\mathrm{J}=3.2 \mathrm{~Hz}), 7.60\left(\mathrm{~d}, 2 \mathrm{H}, \mathrm{H}_{2} \mathrm{Caromatic}, \mathrm{J}=7.5 \mathrm{~Hz}\right)$, $7.69(\mathrm{~d}, 2 \mathrm{H}, \mathrm{HC}$ aromatic, $\mathrm{J}=7.5 \mathrm{~Hz}), 7.72(\mathrm{~d}, 1 \mathrm{H}, \mathrm{HC}$ aromatic, $\mathrm{J}=7.8 \mathrm{~Hz}), 7.76\left(\mathrm{~s}, 1 \mathrm{H}, \mathrm{HN} \mathrm{D} \mathrm{D}_{2} \mathrm{O}\right.$ exchangeable), $8.30\left(\mathrm{~s}, 1 \mathrm{H}, \mathrm{HC}=\mathrm{N}\right.$ aromatic), ${ }^{13} \mathrm{C}$ NMR (DMSO-d $): \delta 77.2(\mathrm{CH}), 125.4(\mathrm{CH})$, 129(CH), 138(CH), 160(CH), MS (m/z,r.i.\%): 338(M+1 $100 \%), 189(23 \%), 163(76 \%)$.

$\mathrm{N}-(2 \mathrm{H}-\mathrm{Benzo}[\mathrm{b}][1,4]$ thiazin-3-yl)-4-methylbenzenesulfonamide(5b): Green solid in $59 \%$ yield, m.p. $=198-199^{\circ} \mathrm{C}, \quad \mathrm{C}_{15} \mathrm{H}_{14} \mathrm{~N}_{2} \mathrm{O}_{2} \mathrm{~S}_{2}(318.41)$, Analysis\% Calcd (Found): C: 56.58(56.60), H:4.43(4.42), N: 8.80(8.79), S: 20.14 (20.11). IR (KBr) $\max / \mathrm{cm}^{-1}: 3403(\mathrm{NH})$, $1310(\mathrm{~S}=\mathrm{O}) .{ }^{1} \mathrm{HNMR}$ (DMSO-d $): \delta 2.39$ (s, 3H, H3 $\mathrm{C}$ ), 4.45(s, 2H, HC), 7.15-7.18(dd, 1H, HC aromatic, $\mathrm{J}=9 \mathrm{~Hz}), 7.32(\mathrm{t}, 1 \mathrm{H}, \mathrm{HC}$ aromatic, $\mathrm{J}=2.1 \mathrm{~Hz}), 7.59\left(\mathrm{~d}, 2 \mathrm{H}, \mathrm{H}_{2} \mathrm{C}\right.$ aromatic, $\left.\mathrm{J}=7.8 \mathrm{~Hz}\right)$, 7.63(d,2H, $\mathrm{HC}$ aromatic, $\mathrm{J}=7.8 \mathrm{~Hz}), 7.72(\mathrm{~d}, 1 \mathrm{H}, \mathrm{HC}$ aromatic, $\mathrm{J}=7.8 \mathrm{~Hz}), 7.76\left(\mathrm{~s}, 1 \mathrm{H}, \mathrm{HN} \mathrm{D}_{2} \mathrm{O}\right.$ exchangeable), 8.12(s,1H, HC=N ), MS (m/z, r.i.\%): 318( $\left.\mathrm{M}^{+}, 100 \%\right), 170(82 \%), 98(21 \%)$.

$\mathrm{N}-(2 \mathrm{H}-$ benzo[b][1,4]thiazin-3-yl)benzenesulfonamide(5c):Brown solid, in $69 \%$ yield, m.p. $=210-211^{\circ} \mathrm{C}, \mathrm{C}_{14} \mathrm{H}_{12} \mathrm{~N}_{2} \mathrm{O}_{2} \mathrm{~S}_{2}$ (304.39), Analysis\% Calcd (Found): C:55.24(55.21), H: 3.79(3.80), N: 9.20(9.22), S: 21.07 (21.08). IR (KBr) $\max / \mathrm{cm}^{-1}: 3325(\mathrm{NH}), 1289$ (S=O). ${ }^{1} \mathrm{H}$ NMR (DMSO-d6):4.42(s, 2H, HC), 7.22-7.29(dd, 1H, HC aromatic, J=6Hz), 7.35(t, 1H, HC aromatic , $\mathrm{J}=2.1 \mathrm{~Hz}), 7.55-7.68(\mathrm{~m}, 5 \mathrm{H}, \mathrm{ArsH}), 7.70(\mathrm{~d}, 1 \mathrm{H}, \mathrm{HC}$ aromatic, $\mathrm{J}=12 \mathrm{~Hz}), 7.76(\mathrm{~s}, 1 \mathrm{H}$, $\mathrm{HN} \mathrm{D}_{2} \mathrm{O}$ exchangeable), 8.25(s,1H, HC=N ), ${ }^{13} \mathrm{C}$ NMR (DMSO-d $): 877.2(\mathrm{CH}), 125.4(\mathrm{CH})$, 129(CH), 138(CH), 158(CH), MS (m/z, r.i.\%): 304(M+1 $100 \%), 156(24 \%), 147(22 \%)$.

4-Chloro-N-(quinoxalin-2-yl)benzenesulfonamide (6a): Brown solid in $71 \%$ yield, m.p. $=184-185^{\circ} \mathrm{C}, \mathrm{C}_{14} \mathrm{H}_{10} \mathrm{ClN}_{3} \mathrm{O}_{2} \mathrm{~S}$ (319.76), Analysis\% Calcd (Found): C: 52.59(52.60), H: 3.15(3.19), Cl: 11.09(11.12), N: 13.14(13.11), S:10.03(10.05). IR (KBr) $\max / \mathrm{cm}^{-1}: 3310(\mathrm{NH})$, $1302(\mathrm{~S}=\mathrm{O}) .{ }^{1} \mathrm{H}$ NMR (DMSO-d $): \delta 7.60\left(\mathrm{~s}, 2 \mathrm{H}, \mathrm{H}_{2} \mathrm{C}\right.$ aromatic, $\left.\mathrm{J}=7.5 \mathrm{~Hz}\right), 7.63(\mathrm{dd}, 1 \mathrm{H}, \mathrm{HC}$ aromatic, $\mathrm{J}=1.8 \mathrm{~Hz}), 7.69(\mathrm{~d}, 2 \mathrm{H}, \mathrm{HC}$ aromatic, $\mathrm{J}=7.5 \mathrm{~Hz}), 7.84(\mathrm{dd}, 2 \mathrm{H}, \mathrm{HC}$ aromatic, $\mathrm{J}=$ $1.2 \mathrm{~Hz}), 8.01(\mathrm{~d}, 1 \mathrm{H}, \mathrm{HC}$ aromatic, $\mathrm{J}=6.2 \mathrm{~Hz}), 9.50(\mathrm{~s}, 1 \mathrm{H}, \mathrm{HC}$ quinoxaline), $11.35(\mathrm{~s}, 1 \mathrm{H}, \mathrm{HN}-$ $\mathrm{D}_{2} \mathrm{O}$ exchangeable). ${ }^{13} \mathrm{CNMR}$ (DMSO-d $): \delta 119.3(\mathrm{CH}), 122.2(\mathrm{CH}), 125.1(\mathrm{CH}), 128.3(\mathrm{CH})$, 131(CH), 156(CH), 178(CH), MS (m/z, r.i.\%): 319(M $\left.\mathrm{M}^{+}, 100 \%\right), 321\left(\mathrm{M}^{+2}, 33 \%\right), 190(21 \%)$.

4-Methyl-N-(quinoxalin-2-yl)benzenesulfonamide (6b): Dark brown solid in 69\% yield, m.p. $=164-165^{\circ} \mathrm{C}, \mathrm{C}_{15} \mathrm{H}_{13} \mathrm{~N}_{3} \mathrm{O}_{2} \mathrm{~S}$ (299.35), Analysis\% Calcd (Found): C: 60.18(60.22), $\mathrm{H}:$ 4.38(4.40), N: 14.04(14.10), S: 10.71(10.73). IR (KBr) $\max / \mathrm{cm}^{-1}: 3310(\mathrm{NH}), 1302(\mathrm{~S}=\mathrm{O}) .{ }^{1} \mathrm{H}$ NMR (DMSO-d6): $\delta$ 2.42(s, 3H, H3C), 7.35(d, 2H, HC aromatic, J=3.2Hz), 7.45(dd, 1H, HC aromatic, $\mathrm{J}=2.4 \mathrm{~Hz}), 7.70(\mathrm{~d}, 2 \mathrm{H}, \mathrm{HC}$ aromatic, $\mathrm{J}=12 \mathrm{~Hz}), 7.80(\mathrm{dd}, 2 \mathrm{H}, \mathrm{HC}$ aromatic, $\mathrm{J}=7.8 \mathrm{~Hz})$, 8.01(d, $1 \mathrm{H}, \mathrm{HC}$ aromatic , J=6Hz), 9.48(s, $1 \mathrm{H}, \mathrm{HC}$ quinoxaline), 11.49(s, $1 \mathrm{H}, \mathrm{HN}-\mathrm{D}_{2} \mathrm{O}$ exchangeable), ${ }^{13} \mathrm{C}$ NMR (DMSO-d $): \delta 19.2\left(\mathrm{CH}_{3}\right), 118.2(\mathrm{CH}), 121.8(\mathrm{CH}), 125.3(\mathrm{CH})$, 129.3(CH), 134(CH), 158(CH), 177.3(CH), MS (m/z,r.i.\%): 299( $\left.\mathrm{M}^{+}, 100 \%\right), 144(39 \%)$, $170(43 \%)$.

$\mathrm{N}$-(Quinoxalin-2-yl)benzenesulfonamide (6c): Brown solid in 66\% yield, m.p. $=162-$ $164^{\circ} \mathrm{C}, \mathrm{C}_{14} \mathrm{H}_{11} \mathrm{~N}_{3} \mathrm{O}_{2} \mathrm{~S}$ (285.32), Analysis\% Calcd(Found): C:58.93(58.95), H: 3.89(3.90), $\mathrm{N}: 14.73(14.78), \mathrm{S}: 11.24$ (11.26). IR (KBr) $\max / \mathrm{cm}^{-1}: 3310(\mathrm{NH}), 1302(\mathrm{~S}=\mathrm{O}) .{ }^{1} \mathrm{H}$ NMR (DMSO-d6): $\delta 7.25(\mathrm{~d}, 2 \mathrm{H}, \mathrm{HC}$ aromatic, $\mathrm{J}=12 \mathrm{~Hz}), 7.33(\mathrm{dd}, 1 \mathrm{H}, \mathrm{HC}$ aromatic, $\mathrm{J}=2.5 \mathrm{~Hz}), 7.55-$ $7.68(\mathrm{~m}, 5 \mathrm{H}, \mathrm{HArs}), 8.00(\mathrm{~d}, 1 \mathrm{H}, \mathrm{HC}$ aromatic, $\mathrm{J}=7.2 \mathrm{~Hz}), 9.13(\mathrm{~s}, 1 \mathrm{H}, \mathrm{HC}$ quinoxaline $)$, 11.52(s, $1 \mathrm{H}, \mathrm{HN}-\mathrm{D}_{2} \mathrm{O}$ exchangeable), MS (m/z, r.i.\%): 285( $\left.\mathrm{M}^{+}, 100 \%\right), 156(45 \%), 144(25 \%)$. 
2.1.4. Reaction of 2-bromo-N-(phenylsulfonyl)acetamidederivatives (1a-c) with pyridin-4amine, benzo[d]thiazole-2-thiol and $1 \mathrm{H}$-benzo[d]imidazole-2-thiol.

A mixture of the 2-bromo-N-(phenylsulfonyl)acetamidederivatives (1a-c) $(0.33 \mathrm{~g}, 2$ mmol), and the pyridin-4-amine, benzo [d]thiazole-2-thiol or 1H-benzo[d]imidazole-2-thiol (2 $\mathrm{mmol})$ in ethanol $(10 \mathrm{ml})$ and a few drops of piperidine were refluxed for 10 hours. The reaction mixture was evaporated in vacuo, and residual solid was collected via filtration, washed with ethanol, dried, and finally recrystallized from the suitable solvent to afford the corresponding heterocyclic derivatives 7a-c, 8a-c, and 9a-c[8] recrystallized from $\left(\mathrm{DMF} / \mathrm{H}_{2} \mathrm{O}\right)$, The physical and spectral data are listed below.

N-(4-Chlorophenylsulfonyl)-2-(pyridin-4-ylamino)acetamide (7a): Orange solidin $77 \%$ yield, m.p. $=166-167^{\circ} \mathrm{C}, \mathrm{C}_{13} \mathrm{H}_{12} \mathrm{ClN}_{3} \mathrm{O}_{3} \mathrm{~S}$ (325.77), Analysis\% Calcd (Found):C: 47.93(47.95), H:3.71(3.76), Cl: 10.88(10.89), N: 12.90(12.88), S: 9.84 (9.86). IR (KBr) $\max / \mathrm{cm}^{-}$ 1: 3425(NH), $3326(\mathrm{NH}), 1632(\mathrm{C}=\mathrm{O}), 1325(\mathrm{~S}=\mathrm{O}) .{ }^{1} \mathrm{H}$ NMR (DMSO-d $)$ : $\delta 3.25\left(\mathrm{~s}, 2 \mathrm{H}, \mathrm{H}_{2} \mathrm{C}\right)$, 4.53(s, 1H, $\mathrm{HN} \mathrm{D}_{2} \mathrm{O}$ exchangeable), 6.9(d, 2H, HC pyridyl, $\left.\mathrm{J}=6.3 \mathrm{~Hz}\right), 7.68(\mathrm{~d}, 2 \mathrm{H}, \mathrm{HC}$ aromatic, $\mathrm{J}=1.2 \mathrm{~Hz}), 7.70(\mathrm{~d}, 2 \mathrm{H}, \mathrm{HC}$ aromatic, $\mathrm{J}=7.8 \mathrm{~Hz}), 8.53(\mathrm{~d}, 2 \mathrm{H}, \mathrm{HC}$ pyridyl, $\mathrm{J}=6.1 \mathrm{~Hz})$, 11.4(s, $1 \mathrm{H}, \mathrm{HN}-\mathrm{D}_{2} \mathrm{O}$ exchangeable). ${ }^{13} \mathrm{C}$ NMR (DMSO-d6): $\delta 56\left(\mathrm{CH}_{2}\right), 107.3(\mathrm{CH}), 129(\mathrm{CH})$, 138(CH), 149(CH), 156(CH), 173(C=O), MS (m/z, r.i.\%): 325( $\left.\mathrm{M}^{+}, 100 \%\right), 327\left(\mathrm{M}^{+2}, 32 \%\right)$, $150(56 \%), 113(21 \%)$.

2-(Pyridin-4-ylamino)-N-tosylacetamide (7b): Dark orange powder in $76 \%$ yield, m.p. $=130-132^{\circ} \mathrm{C}, \mathrm{C}_{14} \mathrm{H}_{15} \mathrm{~N}_{3} \mathrm{O}_{3} \mathrm{~S}$ (305.08), Analysis\% Calcd (Found): C: 55.07(55.10), H: 4.95(4.96), N: 13.76(13.78), S: 10.50 (10.52). IR (KBr) max $/ \mathrm{cm}^{-1}: 3416(\mathrm{NH}), 3307(\mathrm{NH})$, 1621(C=O), $1300(\mathrm{~S}=\mathrm{O}) .{ }^{1} \mathrm{H}$ NMR (DMSO-d $)$ ): 2.21 (s, 3H, H3 $\mathrm{C}$ ), 3.35(s, 2H, $\left.\mathrm{H}_{2} \mathrm{C}\right), 5.02(\mathrm{~s}$, $1 \mathrm{H}, \mathrm{HN} \mathrm{D}_{2} \mathrm{O}$ exchangeable), 7.01(d,2H, $\mathrm{HC}$ pyridyl, $\left.\mathrm{J}=6.3 \mathrm{~Hz}\right), 7.53(\mathrm{~d}, 2 \mathrm{H}, \mathrm{HC}$ aromatic, $\mathrm{J}=$ $1.2 \mathrm{~Hz}), 7.69(\mathrm{~d}, 2 \mathrm{H}, \mathrm{HC}$ aromatic, $\mathrm{J}=3.2 \mathrm{~Hz}), 8.61(\mathrm{~d}, 2 \mathrm{H}, \mathrm{HC}$ pyridyl, $\mathrm{J}=6.1 \mathrm{~Hz}), 12.03(\mathrm{~s}, 1 \mathrm{H}$, $\mathrm{HN}-\mathrm{D}_{2} \mathrm{O}$ exchangeable). ${ }^{13} \mathrm{C}$ NMR (DMSO-d6): $\delta \quad 20.2\left(\mathrm{CH}_{3}\right), 54.3\left(\mathrm{CH}_{2}\right), 107.3(\mathrm{CH})$, $129(\mathrm{CH}), \quad 137.2(\mathrm{CH}), \quad 149(\mathrm{CH}), \quad 154(\mathrm{CH}), \quad 170(\mathrm{C}=\mathrm{O}), \mathrm{MS}\left(\mathrm{m} / \mathrm{z}, \quad\right.$ r.i.\%): 305( $\left.\mathrm{M}^{+}, 100 \%\right)$, $236(23 \%), 170(52 \%), 150(21 \%)$.

$\mathrm{N}$-(Phenylsulfonyl)-2-(pyridin-4-ylamino)acetamide (7c): Orange solid, in 74\% yield, m.p. $=152-154^{\circ} \mathrm{C}, \mathrm{C}_{13} \mathrm{H}_{13} \mathrm{~N}_{3} \mathrm{O}_{3} \mathrm{~S}$ (291.33), Analysis\% Calcd (Found): C: 53.60(53.58), $\mathrm{H}: 4.50(4.48), \mathrm{N}: 14.42(14.46), \mathrm{S}: 11.00(10.89)$. IR (KBr) $\max / \mathrm{cm}^{-1}: 3400(\mathrm{NH}), 3310(\mathrm{NH})$, 1633(C=O), 1289(S=O). ${ }^{1} \mathrm{H}$ NMR (DMSO-d $): \delta 3.31\left(\mathrm{~s}, 2 \mathrm{H}, \mathrm{H}_{2} \mathrm{C}\right), 5.01\left(\mathrm{~s}, 1 \mathrm{H}, \mathrm{HN} \mathrm{D}_{2} \mathrm{O}\right.$ exchangeable), 7.01(d, 2H, $\mathrm{HC}$ pyridyl, $\mathrm{J}=6.3 \mathrm{~Hz}), 7.68(\mathrm{~d}, 2 \mathrm{H}, \mathrm{HC}$ aromatic, $\mathrm{J}=7.8 \mathrm{~Hz}), 7.70$ $(\mathrm{d}, 2 \mathrm{H}, \mathrm{HC}$ aromatic, $\mathrm{J}=3.2 \mathrm{~Hz}), 8.60(\mathrm{~d}, 2 \mathrm{H}, \mathrm{HC}$ pyridyl, $\mathrm{J}=6.1 \mathrm{~Hz}), 12.01\left(\mathrm{~s}, 1 \mathrm{H}, \mathrm{HN}-\mathrm{D}_{2} \mathrm{O}\right.$ exchangeable). MS (m/z, r.i.\%): $291\left(\mathrm{M}^{+}, 100 \%\right), 213(55 \%), 156(12 \%)$.

2-(Benzo[d]thiazol-2-ylthio)-N-(4-chlorophenylsulfonyl)acetamide (8a): Yellow solid in $64 \%$ yield, m.p. $=204-205^{\circ} \mathrm{C}, \mathrm{C}_{15} \mathrm{H}_{11} \mathrm{ClN}_{2} \mathrm{O}_{3} \mathrm{~S}_{3}$ (398.91), Analysis\% Calcd (Found): $\mathrm{C}$ : 45.16(45.20), H: 2.78(2.81), Cl: 8.89(8.90), N: 7.02(7.05), S: 24.11 (24.08), IR(KBr) $\mathrm{max} / \mathrm{cm}^{-1}$ : 3215(NH), 1611(C=O), $1305(\mathrm{~S}=\mathrm{O}) .{ }^{1} \mathrm{H}$ NMR (DMSO-d 6$): \delta 4.51\left(\mathrm{~s}, 2 \mathrm{H}, \mathrm{H}_{2} \mathrm{C}\right), 7.50(\mathrm{~d}, 2 \mathrm{H}$, $\mathrm{H}_{2} \mathrm{C}$ aromatic, $\left.\mathrm{J}=6.1 \mathrm{~Hz}\right), 7.63(\mathrm{~d}, 2 \mathrm{H}, \mathrm{HC}$ aromatic, $\mathrm{J}=6.2 \mathrm{~Hz}), 7.78(\mathrm{~d}, 2 \mathrm{H}, \mathrm{HC}$ aromatic, $\mathrm{J}=$ $7.5 \mathrm{~Hz}), 7.85(\mathrm{~d}, 1 \mathrm{H}, \mathrm{HC}$ aromatic, $\mathrm{J}=3.2 \mathrm{~Hz}), 7.9(\mathrm{~d}, 1 \mathrm{H}, \mathrm{HC}$ aromatic, $\mathrm{J}=1.2 \mathrm{~Hz}), 12.3(\mathrm{~s}, 1 \mathrm{H}$, $\mathrm{HN}-\mathrm{D}_{2} \mathrm{O}$ exchangeable). ${ }^{13} \mathrm{C}$ NMR (DMSO-d $): \delta 38.2\left(\mathrm{CH}_{2}\right), 123(\mathrm{CH}), 124(\mathrm{CH}), 136.2(\mathrm{CH})$, 154(CH), 164(CH),176(C=O), MS (m/z, r.i.\%): 398( $\left.\mathrm{M}^{+}, 100 \%\right), 400\left(\mathrm{M}^{+2}, 31 \%\right), 223(56 \%)$, $189(7 \%), 179(21 \%)$.

2-(Benzo[d]thiazol-2-ylthio)-N-tosylacetamide (8b): Brown solid , in 62\% yield, m.p. $=208-210^{\circ} \mathrm{C}, \mathrm{C}_{16} \mathrm{H}_{14} \mathrm{~N}_{2} \mathrm{O}_{3} \mathrm{~S}_{3}$ (378.49), Analysis\% Calcd (Found): C: 50.78(50.80), H: 3.73(3.75), N: 7.40(7.38), S: 25.41 (25.42). IR (KBr) $\max / \mathrm{cm}^{-1}: 3303(\mathrm{NH}), 1609(\mathrm{C}=\mathrm{O}), 1310$ 
$(\mathrm{S}=\mathrm{O}) .{ }^{1} \mathrm{H}$ NMR (DMSO-d6): $\delta$ 2.37(s, 3H, H3 C), 4.49(s, 2H, $\left.\mathrm{H}_{2} \mathrm{C}\right), 7.39\left(\mathrm{~d}, 2 \mathrm{H}, \mathrm{H}_{2} \mathrm{C}\right.$ aromatic , $\mathrm{J}=6.1 \mathrm{~Hz}), 7.63(\mathrm{~d}, 2 \mathrm{H}, \mathrm{HC}$ aromatic, $\mathrm{J}=3.2 \mathrm{~Hz}), 7.72(\mathrm{~d}, 2 \mathrm{H}, \mathrm{HC}$ aromatic, $\mathrm{J}=7.5 \mathrm{~Hz})$, $7.85(\mathrm{~d}, 1 \mathrm{H}, \mathrm{HC}$ aromatic , J=3.2Hz), 7.92(d, 1H, HC aromatic , J=1.2Hz), 11.89(s, 1H, HN$\mathrm{D}_{2} \mathrm{O}$ exchangeable), MS (m/z,r.i.\%): 378( $\left.\mathrm{M}^{+}, 100 \%\right), 223$ (48\%), 189(5\%).

2-(Benzo[d]thiazol-2-ylthio)-N-(phenylsulfonyl)acetamide (8c): Reddish brown in $59 \%$ yield, m.p. $=225-226^{\circ} \mathrm{C}, \mathrm{C}_{15} \mathrm{H}_{12} \mathrm{~N}_{2} \mathrm{O}_{3} \mathrm{~S}_{3}$ (364.46), Analysis\% Calcd (Found): $\mathrm{C}$ : 49.43(49.45), H: 3.32(3.30), N: 7.69(7.65), S: 26.39 (26.42). IR (KBr) max $/ \mathrm{cm}^{-1}: 3303(\mathrm{NH})$, 1609(C=O), $1310(\mathrm{~S}=\mathrm{O}) .{ }^{1} \mathrm{H}$ NMR (DMSO-d $): \delta 4.59\left(\mathrm{~s}, 2 \mathrm{H}, \mathrm{H}_{2} \mathrm{C}\right), 7.39\left(\mathrm{~d}, 2 \mathrm{H}, \mathrm{H}_{2} \mathrm{C}\right.$ aromatic, $\mathrm{J}=6.1 \mathrm{~Hz}), 7.55-7.68(\mathrm{~m}, 5 \mathrm{H}, \mathrm{ArsH}), 7.85(\mathrm{~d}, 1 \mathrm{H}, \mathrm{HC}$ aromatic, $\mathrm{J}=1.2 \mathrm{~Hz}), 7.9(\mathrm{~d}, 1 \mathrm{H}, \mathrm{HC}$ aromatic , J=3.2Hz), 12.5 (s, $1 \mathrm{H}, \mathrm{HN}-\mathrm{D}_{2} \mathrm{O}$ exchangeable). ${ }^{13} \mathrm{C} \mathrm{NMR}$ (DMSO-d6): $\delta 37.2\left(\mathrm{CH}_{2}\right)$, $123(\mathrm{CH}), \quad 124(\mathrm{CH}), \quad 136.2(\mathrm{CH}), \quad 155(\mathrm{CH}), \quad 168(\mathrm{CH}), \quad 171(\mathrm{C}=\mathrm{O}), \quad \mathrm{MS} \quad(\mathrm{m} / \mathrm{z}, \mathrm{r} . \mathrm{i} . \%)$ : 364( $\left.\mathrm{M}^{+}, 100 \%\right), 223(43 \%), 189(12 \%)$.

2-(1H-Benzo[d]imidazol-2-ylthio)-N-(4-chlorophenylsulfonyl)acetamide (9a): Yellow solid, in $61 \%$ yield, m.p. $=160-161^{\circ} \mathrm{C}, \mathrm{C}_{15} \mathrm{H}_{12} \mathrm{ClN}_{3} \mathrm{O}_{3} \mathrm{~S}_{2}$ (381.86), Analysis $\%$ Calcd (Found): $\mathrm{C}: 47.18(47.20), \mathrm{H}: 3.17(3.18), \mathrm{Cl}: 9.28(9.30), \mathrm{N}: 11.00(10.98), \mathrm{S}: 16.79$ (16.77). IR $(\mathrm{KBr})_{\max } / \mathrm{cm}^{-1}: 3350(\mathrm{NH}), 3210(\mathrm{NH}), 1637(\mathrm{C}=\mathrm{O}), 1310(\mathrm{~S}=\mathrm{O}) .{ }^{1} \mathrm{H}$ NMR (DMSO-d $)$ ): $\delta$ 4.53(s, $\left.2 \mathrm{H}, \mathrm{H}_{2} \mathrm{C}\right), 7.11(\mathrm{~d}, 2 \mathrm{H}, \mathrm{HC}$ aromatic, $\mathrm{J}=3.2 \mathrm{~Hz}), 7.39(\mathrm{~d}, 2 \mathrm{H}, \mathrm{HC}$ aromatic, $\mathrm{J}=7.8 \mathrm{~Hz})$, 7.63(d, $2 \mathrm{H}, \mathrm{HC}$ aromatic, $\mathrm{J}=7.5 \mathrm{~Hz}), 7.74(\mathrm{~d}, 2 \mathrm{H}, \mathrm{HC}$, aromatic , J=7.8Hz), 11.8(s, 1H, HN $\mathrm{D}_{2} \mathrm{O}$ exchangeable), $12.2\left(\mathrm{~s}, 1 \mathrm{H}, \mathrm{HN}\right.$ imidazole $\mathrm{D}_{2} \mathrm{O}$ exchangeable). ${ }^{13} \mathrm{C}$ NMR (DMSO- $\left.\mathrm{d}_{6}\right): \delta$ 38.1 $\left(\mathrm{CH}_{2}\right), 115.2(\mathrm{CH}), 123(\mathrm{CH}), 138(\mathrm{CH}), 148(\mathrm{CH}), 170(\mathrm{C}=\mathrm{O}), \mathrm{MS}(\mathrm{m} / \mathrm{z}, \mathrm{r} . \mathrm{i} . \%): 381\left(\mathrm{M}^{+}\right.$, $100 \%), 383\left(\mathrm{M}^{+2}, 33 \%\right), 206(10 \%), 189(24 \%)$.

2-(1H-Benzo[d]imidazol-2-ylthio)-N-tosylacetamide (9b): Pale yellow, in $62 \%$ yield,m.p. $=185-186^{\circ} \mathrm{C}, \mathrm{C}_{16} \mathrm{H}_{15} \mathrm{~N}_{3} \mathrm{O}_{3} \mathrm{~S}_{2}(361.44)$, Analysis\% Calcd (Found): C: $53.17(53.20), \mathrm{H}$ : 4.18(4.15), N: 11.63(11.69), S: 17.74 (17.76). IR (KBr) $\max / \mathrm{cm}^{-1}: 3350(\mathrm{NH}), 3210(\mathrm{NH})$, 1613(C=O), $1310(\mathrm{~S}=\mathrm{O}) .{ }^{1} \mathrm{H}$ NMR (DMSO-d6): $\delta$ 2.39(s, 3H, $\left.\mathrm{H}_{3} \mathrm{C}\right), 7.13(\mathrm{~d}, 2 \mathrm{H}, \mathrm{HC}$ aromatic, $\mathrm{J}=1.2 \mathrm{~Hz}), 7.40(\mathrm{~d}, 2 \mathrm{H}, \mathrm{HC}$ aromatic, $\mathrm{J}=6 \mathrm{~Hz}), 7.52(\mathrm{~d}, 2 \mathrm{H}, \mathrm{HC}$ aromatic, $\mathrm{J}=6 \mathrm{~Hz}), 7.68(\mathrm{~d}, 2 \mathrm{H}$, $\mathrm{HC}$ aromatic, $\mathrm{J}=7.8 \mathrm{~Hz}), 11.8\left(\mathrm{~s}, 1 \mathrm{H}, \mathrm{HN}-\mathrm{D}_{2} \mathrm{O}\right.$ exchangeable $), 12.2\left(\mathrm{~s}, 1 \mathrm{H}, \mathrm{HN}\right.$ imidazole $\mathrm{D}_{2} \mathrm{O}$ exchangeable). ${ }^{13} \mathrm{C}$ NMR (DMSO- $\left.\mathrm{d}_{6}\right): \delta 21.3\left(\mathrm{CH}_{3}\right), \quad 38.2\left(\mathrm{CH}_{2}\right), 115.2(\mathrm{CH}), 123(\mathrm{CH})$, 138(CH), 148(CH), 170(C=O), MS (m/z,r.i.\%): 361( $\left.\mathrm{M}^{+}, 100 \%\right), 206(23 \%), 170(6 \%)$.

2-(1H-Benzo[d]imidazol-2-ylthio)-N-(phenylsulfonyl)acetamide (9c): yellow solid , in $59 \%$ yield, m.p. $=187-188^{\circ} \mathrm{C}, \mathrm{C}_{15} \mathrm{H}_{13} \mathrm{~N}_{3} \mathrm{O}_{3} \mathrm{~S}_{2}$ (347.41), Analysis\% Calcd (Found): C: 51.86(51.88), H: 3.77(3.78), N: 12.10(12.09), S: 18.46(18.49). IR (KBr) $\max / \mathrm{cm}^{-1}: 3350(\mathrm{NH})$, $3210(\mathrm{NH}), 1609(\mathrm{C}=\mathrm{O}), 1310(\mathrm{~S}=\mathrm{O}) .{ }^{1} \mathrm{H}$ NMR (DMSO-d6): $\delta 4.53\left(\mathrm{~s}, 2 \mathrm{H}, \mathrm{H}_{2} \mathrm{C}\right), 7.09(\mathrm{~d}, 2 \mathrm{H}, \mathrm{HC}$ aromatic, $\mathrm{J}=1.2 \mathrm{~Hz}), 7.55-7.68(\mathrm{~m}, 5 \mathrm{H}, \mathrm{ArsH}), 7.74(\mathrm{~d}, 2 \mathrm{H}, \mathrm{HC}$, aromatic, $\mathrm{J}=7.8 \mathrm{~Hz}), 11.8(\mathrm{~s}$, $1 \mathrm{H}, \mathrm{HN}-\mathrm{D}_{2} \mathrm{O}$ exchangeable), $12.2\left(\mathrm{~s}, 1 \mathrm{H}, \mathrm{HN}\right.$ imidazole $\mathrm{D}_{2} \mathrm{O}$ exchangeable). $\mathrm{MS}(\mathrm{m} / \mathrm{z}, \mathrm{r} . \mathrm{i} . \%)$ : 347( $\left.\mathrm{M}^{+}, 100 \%\right), 206(33 \%), 156(6 \%)$.

\subsection{Computational studies.}

Calculations of DFT with a hybrid functional B3LYP (Becke's three-parameter hybrid functional utilizing the BLYP functional correlation with the 6-31G(d) theory level via Berny method [36], were designated using the Gaussian 09W program [37]. No symmetry constraints were applied during the geometry optimization. The wide-ranging assignments of the vibrational modes were accomplished based on the Potential Energy Distribution (PED), calculated using the Vibrational Energy Distribution Analysis (VEDA) program [38]. 


\subsection{Antimalarial activities.}

Sulphonamide compounds were examined in identical on one occasion against chloroquine (CQS) strain and P.falciparum (D10). Incessant in vitro philosophies of sexual erythrocyte stages of $\mathrm{P}$. falciparum were supported through the adapted procedure of Trager and Jensen. Quantitative analysis of the antiplasmodial activity in vitro was examined through the parasite lactate dehydrogenase assay utilizing modified theory designated via Makler:Sulphonamides were equipped with a $2 \mathrm{mg} / \mathrm{ml}$ stock solution in a $10 \%$ DMSO and sonicated to improve solubility. Compounds were examined as a suspension if not melted. Stock solutions were kept at $-20^{\circ} \mathrm{C}$. Additional dilutions were ready on the day of the experiment. Chloroquine (CQ) was recycled as the reference drug. Full dose-response was examined for sulphonamides to measure the inhibition concentration $50 \%$ of parasite growth ( $\mathrm{IC}_{50}$ and $\mathrm{IC}_{90}$ ).

Sulphonamides were examined at a starting concentration of $100 \mu \mathrm{g} / \mathrm{ml}$, which was then successively diluted 2-fold in complete medium to afford ten concentrations, with the lowest concentration being $0.2 \mu \mathrm{g} / \mathrm{ml}$. A similar dilution method was recycled for sulphonamides. CQ was examined at a starting concentration of $100 \mathrm{mg} / \mathrm{ml}$. The highest concentration of solvent to which the parasites were exposed had no measurable effect on the parasite viability (data not shown). The $\mathrm{IC}_{50}$ values were found utilizing nonlinear doseresponse curve fitting analysis via Graph Pad Prism v.4.0 software. The consequences of this biological study are displayed in Table 2.

The MTT-assay is used as a colorimetric assay for cellular growth and survival and compares well with other available assays. The tetrazolium salt MTT was utilized to the extent of all growth and chemosensitivity. The sulphonamide was examined in triplicate on one occasion. Sulphonamides were examined to a $2 \mathrm{mg} / \mathrm{ml}$ stock solution in $10 \%$ methanol or $10 \%$ DMSO and were examined as a suspension if not properly dissolved. Test compounds were stored at $-20{ }^{\circ} \mathrm{C}$ until use. Emetine was used as the reference drug in all experiments. The first concentration of emetine was $100 \mu \mathrm{g} / \mathrm{ml}$, which was successively diluted in a complete medium with 10-fold dilutions to give six concentrations, the lowest being $0.001 \mu \mathrm{g} / \mathrm{ml}$. The same dilution technique was applied to all the test samples. The highest concentration of solvent to which the cells were exposed had no measurable effect on the cell viability (data not shown). The 50\% inhibitory concentration ( $\mathrm{IC}_{50}$ ) values were obtained from full dose-response curves, utilizing a nonlinear dose-response curve fitting analysis via Graph Pad Prism v.4.0 software.

\subsection{Molecular docking.}

The molecular perfect of innovative sulfonamide derivatives was invented utilizing standard bond lengths and angles with the Auto DockVina and noticed by Discovery Studio Client (version 4.2). Subsequent geometry optimization, a systematic conformational examination was supported out to an RMS gradient of $0.01 \AA$, with energy minimization of the resultant conformations employing the Confirmation Examination module implemented in Auto Dock Vina. The Wild-type Plasmodium falciparum dihyd rofolatereductase-thymidylate synthase (PfDHFR-TS) complexed with WR99210, NADPH, and dUMP(PDBID:1j3i). The crystal structure of Pfg 27 from Plasmodium falciparum ( PDBID:1n81). Crystal structure of main protease COVID-19 complex with an inhibitor $\mathrm{N}_{3}$ (PDB ID:6lu7) and closed structure of the (SARS-CoV-2 spike glycoprotein) (PDBID:6vxx) Missing hydrogens was extra to the enzyme, and partial charges were measured. After eliminating the co-crystallized inhibitor, 
validation checked through docking of the compounds was carried out by Auto DockVina and observed through Discovery Studio Client (version 4.2).

The target protein was kept inflexible, although the ligands were disappeared allowable to regulate the conformational space exclusive the enzyme cavity; Twenty discrete docking simulations were run via avoidance parameters, and the confirmations were designated constructed on the arrangement of total statistics, E conformation, and appropriate with the relevant amino acids in the binding pocket.

\section{Results and Discussion}

Nitrogen nucleophiles reaction with Bromo- $N$-(phenylsulfonyl)acetamide derivatives 1a-c. was accomplished through means of Schotten-Baumann reaction[8] as displayed in Scheme 1.

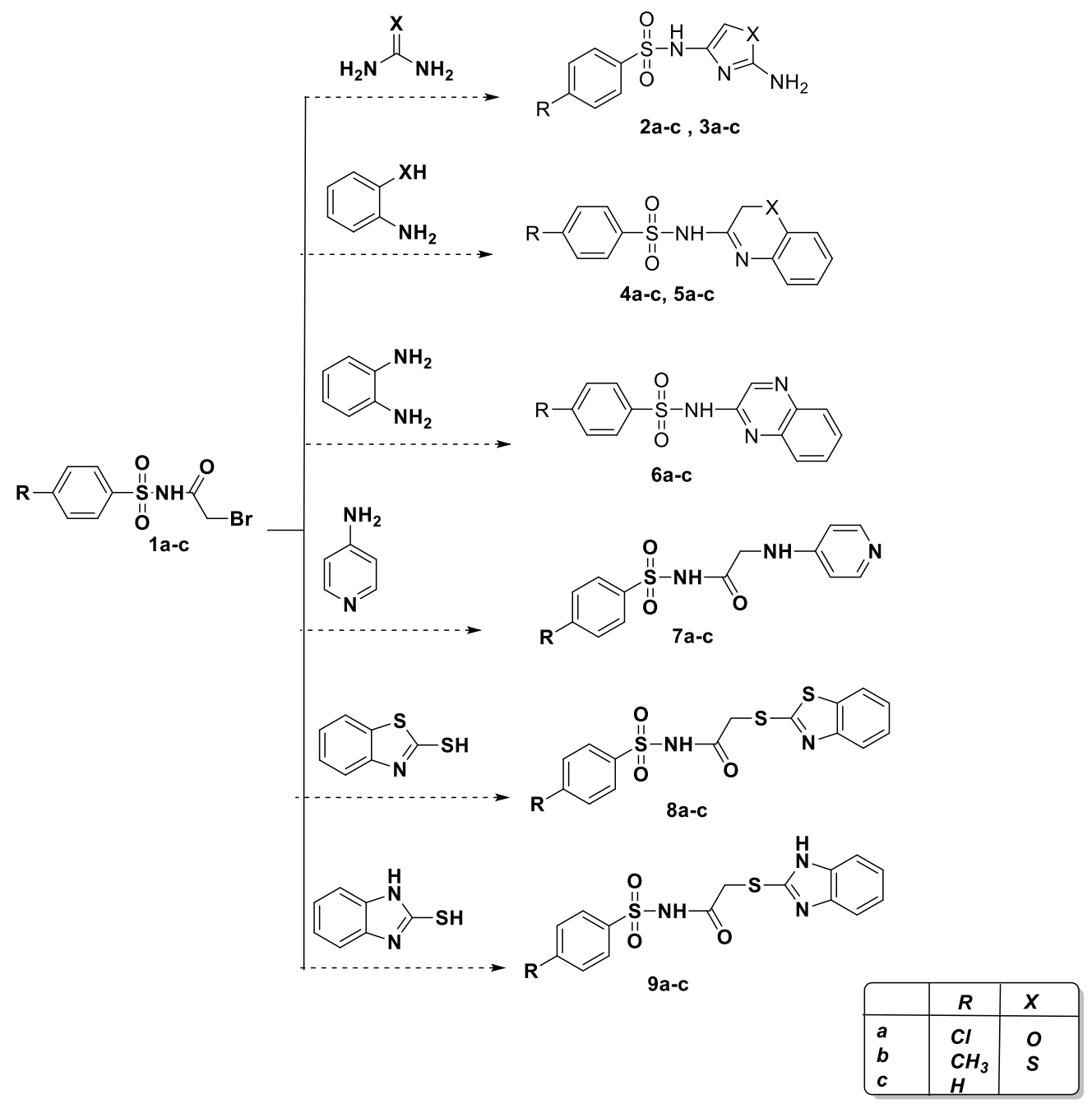

Scheme 1. Synthesis of several sulfonamide from bromo- $N$-(phenylsulfonyl)acetamide 1a-c with different nitrogen nucleophiles.

The performance of the acetamide derivatives 1a-c towards urea derivatives was examined. Therefore, the corresponding aminothiazole derivatives $2 \mathrm{a}-\mathrm{c}$ and aminooxazole derivatives 3a-c, respectively; (Scheme 1) was confirmed via spectral data; for example, the IR spectra of compound $2 \mathrm{~b}$, taken as an example; showed absorption band at $3215 \mathrm{~cm}^{-1}$ due to $\mathrm{NH}$ function, at $3100-3025 \mathrm{~cm}^{-1}$ due to $\mathrm{NH}_{2}$ group and a strong stretching absorption band of the $\mathrm{S}=\mathrm{O}$ group at $1289 \mathrm{~cm}^{-1}$. The ${ }^{1} \mathrm{H}$ NMR spectrum of the same compound revealed a singlet signal at $\delta 6.56$ due to the thiazole proton, at $\delta 10.29$ due to $\mathrm{NH}$ proton, and at $\delta 4.65\left(\mathrm{D}_{2} \mathrm{O}-\right.$ 
exchangeable) due to exchangeable $\mathrm{NH}_{2}$ protons. Its mass spectrum revealed a peak at $\mathrm{m} / \mathrm{z}=$ 269 , corresponding to its molecular ion.

Similarly, compound $3 a$ revealed a singlet signal at $\delta 7.12$ due to the (oxazole- $H C$ ) and singlet signal at 10.25 due to $\mathrm{NH}$ proton. Its ${ }^{13} \mathrm{C}$ NMR spectrum showed a characteristic signal of $\delta \mathrm{CH}$ at $126 \mathrm{ppm}$ due to the oxazole ring. The same compound's IR spectrum revealed the occurrence of two strong absorption bands at $3230-3125 \mathrm{~cm}^{-1}$ corresponding to $\mathrm{NH}_{2}$ group.

Ring-forming reaction of bromo- $N$-(phenylsulfonyl)acetamide derivatives $1 \mathrm{a}-\mathrm{c}$ with heterocycles nitrogen nucleophiles to afford benzo[b][1,4]oxazin-3-yl 4a-c, $2 \mathrm{H}$ benzo[b][1,4]thiazin-3-yl 5a-c and quinoxalin-2-yl 6a-c derivatives, respectively. The reaction afforded in each case, only one isolable product as shown via TLC analysis with different yield as depicted in Scheme 1.

The IR spectra of compound 4c, taken as an example; gave an absorption band at 3320 $\mathrm{cm}^{-1}$ due to NH function. Its ${ }^{1} \mathrm{H}$ NMR revealed signals at $\delta 6.44$ and at $\delta 8.15$ due to oxazin-3$\mathrm{yl}$ and $H \mathrm{C}=\mathrm{N}$ proton, respectively, besides an aromatic multiplied in the region $\delta 7.55-7.68$, Its mass spectrum revealed a peak at $m / z 288$ corresponding to its molecular ion. Furthermore, compound 5a displayed in its ${ }^{1} \mathrm{HNMR}$ spectrum characteristic signals at $\delta 5.33$ and 8.30 due to thiazin-3-yl and $H C=\mathrm{N}$ protons and the ${ }^{13} \mathrm{C} \mathrm{NMR}$ of the same compound showed a characteristic signal of thiazin-3-yl at $\delta 77.2$; and the ${ }^{1} \mathrm{H}$ NMR spectrum of compound $6 \mathrm{~b}$ displayed the singlet signal due to quinoxaline ring at $\delta 9.48$, and Its mass spectrum revealed a peak at $m / z=299$ due to its molecular ion (Scheme 1).

Therefore, when ethanolic compounds 1a-c were refluxed to react with 4aminopyridine in the presence of piperidine to afford the corresponding (sulfonyl)-2-(pyridin4-ylamino)acetamide derivatives 7a-c. The ${ }^{1}$ HNMR spectrum of 7 a showed a signal duo to the active methylene protons at $\delta 3.25$, and the signal appeared at 8.53 due to protons of pyridine moiety. Also, its ${ }^{13} \mathrm{C}$ NMR spectrum showed a signal at $\delta 56$ due to methylene and $\delta 173$ due to carbonyl carbon, as displayed in Scheme 1.

The acetamide derivatives 1a-c were reacted with benzo-2-thiol derivatives to give the corresponding 2-(benzo[d]thiazol-2-ylthio) derivatives 8a-c and 2-(1H-benzo $[d]$ imidazol-2ylthio) 9a-c, respectively; as presented in Scheme 1. For instance, FT-IR spectra of compound $8 \mathrm{c}$ revealed absorption bands due to $\mathrm{NH}$ and sulphoxide functions at $3303(\mathrm{NH})$ and 1310 $(\mathrm{S}=\mathrm{O})$, respectively. Furthermore, its ${ }^{1} \mathrm{H}$ NMR spectrum revealed an aromatic multiplet in the region $\delta 7.55-7.69$, in addition to singlet at $\delta 4.59$ due to $=\mathrm{CH}$ group and at $\delta 12.5 \mathrm{ppm}$ due to proton of $\mathrm{NH}$ [ $c f$. Experimental part] (Scheme 1).

\subsection{Computational calculations:}

\subsubsection{Geometrical Calculations.}

Optimization of 4a, 5c, and 6a compounds utilizing Gaussian program 09 as demonstrated in (Figure 2), and calculated the total energy (Ет), E(номо), E(LUMO), energy gap $\left(\mathrm{E}_{\mathrm{g}}\right)$, dipole moment $(\mu)$, absolute electronegativities $(\chi)$, chemical potentials $(\mathrm{Pi})$, absolute hardness, $(\eta)$ absolute softness $(\sigma)$, global electrophilicity $(\omega)$, global softness (S), and $\left(\Delta \mathrm{N}_{\max }\right)$ additional electronic charge, were listed in Table 1 permitting to the subsequent equations and optimized via DFT/B3LYP/6-31G(d). Nonplanarity of the molecular structure of these compounds. The obtainable potential activities in the originator compound $4 \mathrm{a}, 5 \mathrm{c}$, and $6 a$ due to the fused heterocyclic involved into the sulfonamide ring. 


$$
\begin{aligned}
& \Delta E=E_{\text {LUMO }}-E_{\text {HOMO }} \\
& \eta=\frac{\left(E_{L U M O}-E_{\text {HOMO }}\right)}{2} \\
& \mathrm{Pi}=-\mathrm{X} \\
& \omega=\mathrm{Pi}^{2} / 2 \eta
\end{aligned}
$$

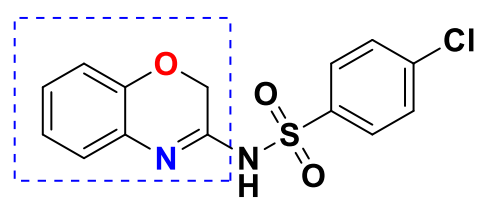

$4 a$
(1) $\chi=\frac{-\left(E_{H O M O}+E_{L U M O}\right)}{2}(2)$

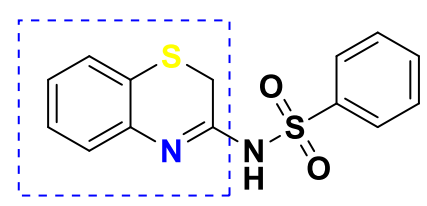

$5 c$

$$
\begin{aligned}
& \sigma=1 / \eta \\
& S=1 / 2 \eta \\
& \Delta \mathrm{N} \max =-\mathrm{Pi} / \eta(8)
\end{aligned}
$$

\begin{tabular}{|c|c|c|c|c|c|c|}
\hline Compound & \multicolumn{2}{|c|}{$4 \mathbf{a}$} & \multicolumn{2}{|c|}{$5 c$} & \multicolumn{2}{|c|}{$\mathbf{6 a}$} \\
\hline$E_{\mathrm{T}}(\mathrm{au})$ & \multicolumn{2}{|c|}{-1733.13} & \multicolumn{2}{|c|}{-1596.571} & \multicolumn{2}{|c|}{-1712.114} \\
\hline Еномо & \multicolumn{2}{|c|}{-0.00344} & \multicolumn{2}{|c|}{-0.00059} & \multicolumn{2}{|c|}{-0.01486} \\
\hline ELUMO $_{\text {Lu }}$ & \multicolumn{2}{|c|}{0.02618} & \multicolumn{2}{|c|}{0.03156} & \multicolumn{2}{|c|}{0.00371} \\
\hline$\Delta \mathbf{E}$ & \multicolumn{2}{|c|}{0.80600} & \multicolumn{2}{|c|}{0.87484648} & \multicolumn{2}{|c|}{0.50531568} \\
\hline$\mu$ (Debye) & \multicolumn{2}{|c|}{5.1792} & \multicolumn{2}{|c|}{5.2473} & \multicolumn{2}{|c|}{4.9617} \\
\hline$\chi(\mathrm{eV})$ & \multicolumn{2}{|c|}{-0.309393607} & \multicolumn{2}{|c|}{-0.34830591} & \multicolumn{2}{|c|}{0.151621915} \\
\hline$\eta(\mathrm{eV})$ & \multicolumn{2}{|c|}{0.40300082} & \multicolumn{2}{|c|}{0.51048585} & \multicolumn{2}{|c|}{0.25265784} \\
\hline$\sigma(e V)$ & \multicolumn{2}{|c|}{1837.3654} & \multicolumn{2}{|c|}{1450.476} & \multicolumn{2}{|c|}{2930.68128} \\
\hline$P_{i}(e V)$ & \multicolumn{2}{|c|}{0.30939361} & \multicolumn{2}{|c|}{0.3483059} & \multicolumn{2}{|c|}{-0.1516219152} \\
\hline $\mathrm{S}(\mathrm{eV})$ & \multicolumn{2}{|c|}{918.6568} & \multicolumn{2}{|c|}{725.24909} & \multicolumn{2}{|c|}{1465.334} \\
\hline$\omega(\mathrm{eV})$ & \multicolumn{2}{|c|}{0.000026032} & \multicolumn{2}{|c|}{0.00004163344047} & \multicolumn{2}{|c|}{0.00000392215} \\
\hline$\Delta \mathbf{N}_{\max }$ & & 724510544 & & 6412 & & 77008 \\
\hline Net Charges & $\mathrm{O}_{12}$ & -0.533 & $\mathrm{~S}_{22}$ & 0.389 & $\mathrm{~N}_{14}$ & -0.367 \\
\hline & $\mathrm{C}_{17}$ & -0.245 & $\mathrm{C}_{17}$ & -581 & $\mathrm{C}_{13}$ & -0.001 \\
\hline & $\mathrm{N}_{15}$ & -0.509 & $\mathrm{~N}_{19}$ & -0.469 & $\mathrm{~N}_{11}$ & -0.433 \\
\hline & $\mathrm{C}_{16}$ & 0.505 & $\mathrm{C}_{18}$ & -0.547 & $\mathrm{C}_{12}$ & 0.524 \\
\hline & $\mathrm{N}_{10}$ & -0.865 & $\mathrm{~N}_{23}$ & -0.871 & $\mathrm{~N}_{10}$ & -0.931 \\
\hline & $\mathrm{C}_{17}$ & -0.045 & $\mathrm{C}_{21}$ & -0.297 & $\mathrm{C}_{15}$ & 0.132 \\
\hline
\end{tabular}<smiles>O=S(=O)(Nc1cnc2ccccc2n1)c1ccc(I)cc1</smiles>

$6 a$

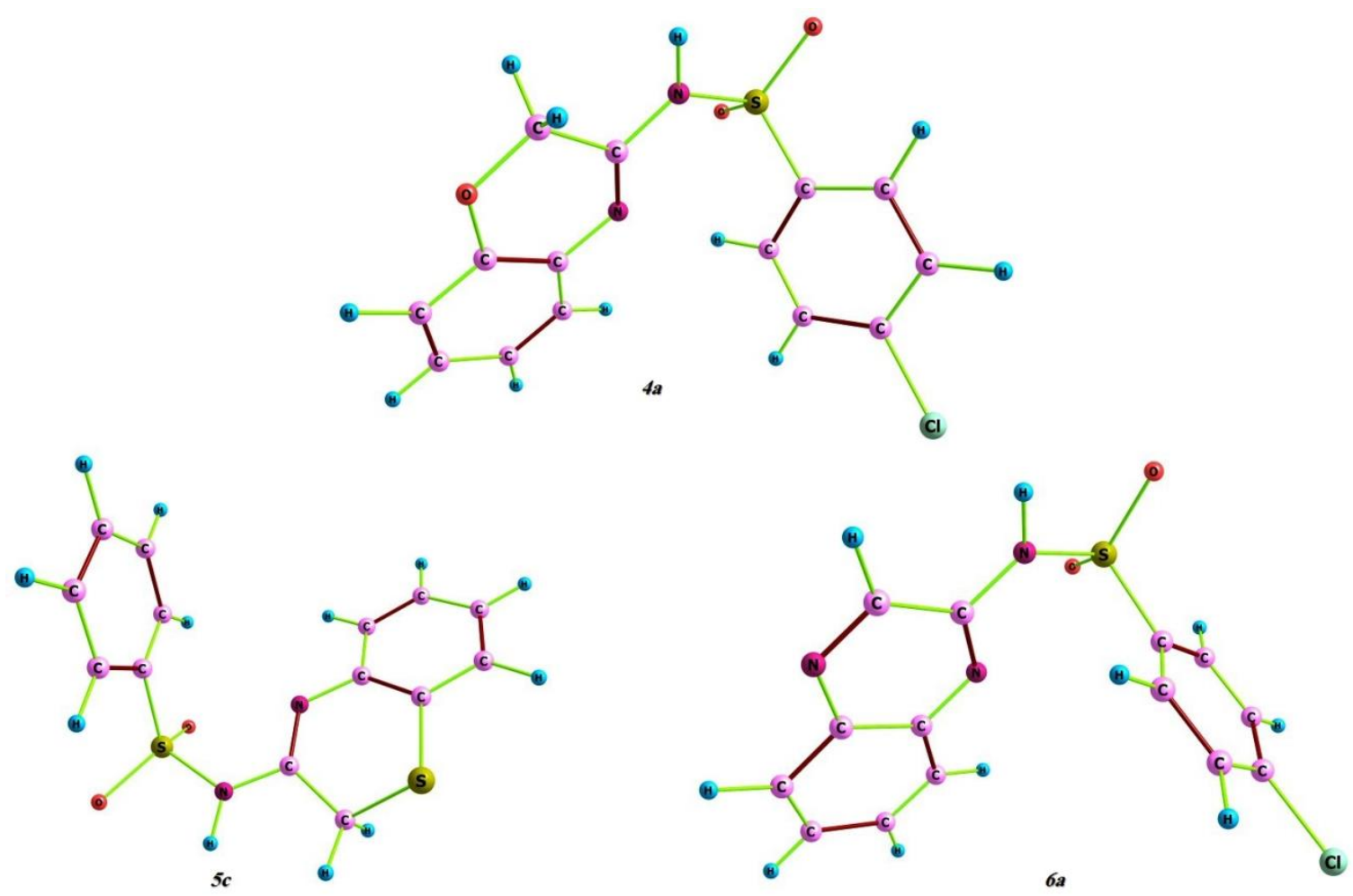

Figure 2. Optimized geometry, numbering system of Compound 4a, 5c, and 6a in DFT/B3LYP/6-31G.

Table 1. Quantum chemical parameters of compounds 4a, 5c, and 6a utilizing DFT/B3LYP/6-31G (d). DFT B3LYP/6-31G(d) 
The outcomes from Tables 1 and Figure 2 the following conclusions were concluded: (1) The optimization of $N$-(2H-benzo[b][1,4] oxazin-3-yl)-4-chlorobenzenesulfonamide (4a), $N$-(2H-benzo[b][1,4]thiazin-3-yl)benzenesulfonamide (5c) and 4-chloro- $N$-(quinoxalin-2yl)benzenesulfonamide (6a) utilizing Gaussian 09 program, indicated that these compounds were non-planer as shown in Figure 2.

(2) The Energy gap ( $E g$ ) of $4 \mathrm{a}$ and $5 \mathrm{c}$ was found to approximately similar in energy, and compound $6 \mathrm{a}$ is the most active compound with the difference in energy $(0.50531568 \mathrm{e}$ $\mathrm{V})(\approx 11.65285 \mathrm{kcal} / \mathrm{mol})$.

(3) These energy difference due to benzo[b][1,4]oxazin-3-yl, benzo[b][1,4]thiazin-3-yl, and quinoxalin-2-yl which attached to sulfonamide ring and from result show that the quinoxaline ring has less energy difference which gives more activity and high biological activity

(4) The absolute electronegativity $(\chi)$ which concept that designates the affinity of an atom to appeal to a mutual pair of electrons, the value of $(\chi)$ for 4aand 5 chave $(-0.309393607 \mathrm{e} V)(\approx-$ $7.13478 \mathrm{kcal} / \mathrm{mol}),(-0.34830591 \mathrm{e} \mathrm{V})(\approx--8.03212 \mathrm{kcal} / \mathrm{mol})$; respectively. While compounds $6 \mathrm{a}$ take the positive value at $(0.151621915 \mathrm{e} \mathrm{V})(\approx 3.4964835 \mathrm{kcal} / \mathrm{mol})$, these values indicate the stability of these compounds and the tendency to react again.

(5) The absolute hardness $(\eta)$ measures the tendency of change in electron density around the molecule, showed that the electron density of $\mathrm{N}-(2 \mathrm{H}-\mathrm{benzo}[\mathrm{b}][1,4]$ thiazin-3yl)benzenesulfonamide $(5 \mathrm{c})$ is more than compounds $4 \mathrm{a}$ and $6 \mathrm{a}$ by $(0.10748585 \mathrm{e}$ $\mathrm{V})(\approx 2.4786 \mathrm{kcal} / \mathrm{mol})$ and $(0.25783585 \mathrm{e} \mathrm{V})(\approx 5.9458 \mathrm{kcal} / \mathrm{mol})$ due to the presence of sulfur atom in benzo[b][1,4]thiazin-3-yl ring which increases its electron density.

(6) Furthermore, the absolute softness $(\sigma)$ indicates the interaction of compound, which showed that compound 6a has high interaction energy via $(2930.68128 \mathrm{e} \mathrm{V})($ $\approx 67583.09 \mathrm{kcal} / \mathrm{mol}$ ) due to the occurrence of the nitrogen atom in the $\mathrm{N}$-(quinoxalin-2-yl), which increase its reactivity.

\subsubsection{FMO (Frontier molecular orbitals).}

FMO is an influential guide for the electrical and optical character; moreover, UV spectral data and chemical reactions were studied TD-DFT (Time-dependent density functional theory). HOMO(highest occupied molecular orbital) which an electron donor, and LUMO (lowest unoccupied molecular orbital) which an electron acceptor. Gap energy between HOMO and LUMO is associated with the biological activity of the molecule. Likewise, it helped in characterize the reactivity and kinetic stability of the compound. HOMO-LUMO's highest gap energy imitates the high kinetic stability [56]. Figure 3 elucidates the spreading and energy altitudes of the HOMO-LUMO and orbitals calculated via the B3LYP/6-31G (d) basis set for $4 \mathrm{a}, 5 \mathrm{c}$, and $6 \mathrm{a}$. The positive and negative points were signified in red and green colors, respectively. As presented in Figure 3, the HOMO of compound 4a localized on the benzo[b][1,4]oxazin-3-yl and LUMO in both benzooxazine and $p$-chlorobenzene ring. The worth of the energy gap between the HOMO and LUMO is $0.800 \mathrm{eV}$ which designates the high excitation energies for frequent excited states and reactivity of this compound.

Similarly, the compound 5c, the HOMO restricted on benzo[b][1,4]thiazin-3-yl and its LUMO originate in both benzene and benzothiazine ring and difference in energy $0.87 \mathrm{eV}$, and lastly, the compound 6a establish its HOMO-LUMO in quinoxalin-2-yl ring with the lowest difference in energy 0. $505 \mathrm{eV}$ as displayed in Figure 3. From the below figure and small HOMO-LUMO energy gap, which certificate that transfer of electrons due to the presence of $\mathrm{O}, \mathrm{N}, \mathrm{S}$ in the fused rings which has electronegativity sites. 

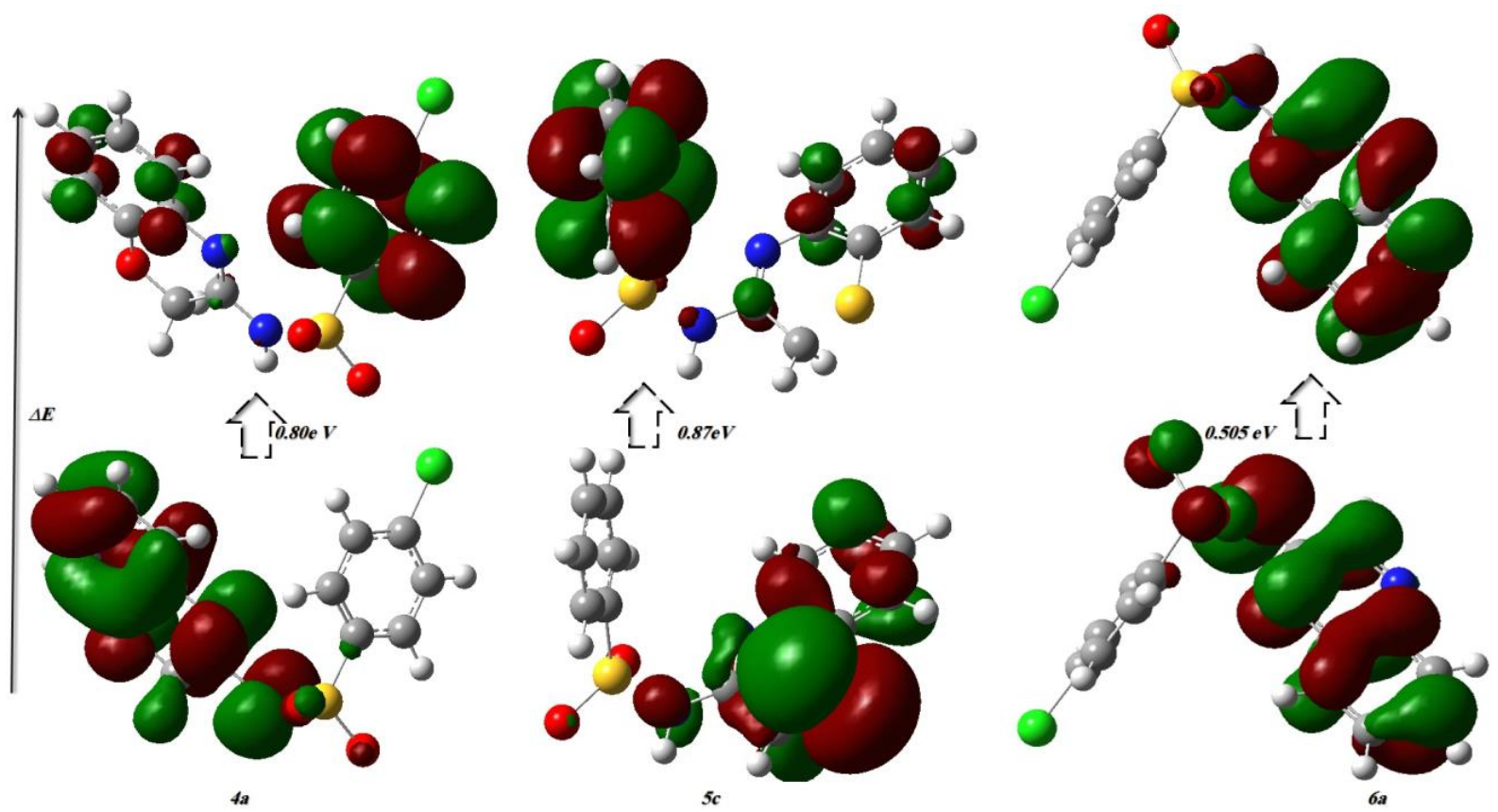

Figure 3. Energy gap (HOMO-LUMO) of compounds 4a, 5c, and 6a utilizing DFT/6-31(G)d basis set.

\subsection{Biological investigation.}

3.2.1. Antiplasmodial activity.

Consequently, 2-Bromo- $N$-(phenylsulfonyl) acetamide, aminothiazole 2a-c, amino oxazole 3a-c, benzo[b][1,4]oxazin-3-yl 4a-c, $2 H$-benzo[b][1,4]thiazin-3-yl 5a-c, quinoxalin2-yl 6a-c, pyridin-4-ylamino 7a-c, benzo $[d]$ thiazol-2-ylthio 8a-c and $1 H$-benzo $[d]$ imidazol-2ylthio 9a-c derivatives were partitioned into antiplasmodial activity.

Table 2. In vitro Antiplasmodial activity against P. Falciparum (CQS) (D10).

\begin{tabular}{|c|c|c|c|c|c|}
\hline Compound & $\mathrm{IC}_{50(\mu \mathrm{M})}$ & $\mathrm{IC}_{90}(\mu \mathrm{M})$ & Compound & $\mathrm{IC}_{50(\mu \mathrm{M})}$ & $\mathrm{IC}_{90(\mu \mathrm{M})}$ \\
\hline $\mathbf{2 a}$ & 33.2 & 65.2 & 6b & 11.3 & $\mathrm{ND}^{a}$ \\
\hline $2 \mathrm{~b}$ & 24.2 & 53.2 & $6 c$ & 27.3 & 38.23 \\
\hline $2 c$ & 44.2 & 63.2 & $7 \mathbf{a}$ & 80.23 & 110.2 \\
\hline $\mathbf{3 a}$ & 45.89 & 154.73 & $7 b$ & 9.41 & 25.2 \\
\hline $\mathbf{3 b}$ & 62.57 & ND & $7 \mathrm{c}$ & 7.23 & 123.3 \\
\hline $3 c$ & 5.32 & 135.22 & $8 \mathbf{a}$ & 45.27 & ND \\
\hline $4 a$ & 11.25 & 29.22 & $8 b$ & 27.23 & 77.23 \\
\hline $4 \mathrm{~b}$ & 24.25 & ND & $8 \mathrm{c}$ & 33.2 & 98.23 \\
\hline $4 c$ & 16.71 & 118.46 & $9 a$ & 12.3 & 56.3 \\
\hline $5 a$ & 3.2 & 6.4 & $9 \mathrm{~b}$ & 75.2 & ND \\
\hline $5 b$ & 30.21 & ND & $9 \mathrm{c}$ & 11.23 & ND \\
\hline $5 c$ & 5.23 & 10.23 & $6 a$ & 1.2 & 6.3 \\
\hline $\operatorname{CQ}(n=8)^{b}$ & 0.017 & 0.067 & $\operatorname{CQ}(n=8)^{b}$ & 0.017 & 0.067 \\
\hline
\end{tabular}

Stimulatingly, these consequences indication that micromolar potency for almost compounds, pointing to the significant biological potential of aminothiazole 2a-c, aminooxazole 3a-c, benzo[b][1,4]oxazin-3-yl 4a-c, $2 H$-benzo[b][1,4]thiazin-3-yl 5a-c, quinoxalin-2-yl 6a-c, pyridin-4-ylamino 7a-c, benzo $[d]$ thiazol-2-ylthio $8 \mathrm{a}-\mathrm{c}$ and $1 \mathrm{H}$ benzo $[d]$ imidazole-2-ylthio 9a-c derivatives. Additionally, 24 compounds demonstrated antimalarial activity with $\mathrm{IC}_{50}$ values of $<30 \mu \mathrm{M}$ against $P$. Falciparum $(C Q S)$ D10 strain, and compounds 3c, 4a, 5a, 6a, 6b, 7b, 7c, 9a, and 9c presented promising biological activity with IC $_{50}$ values $\leq 15 \mu \mathrm{M}$. quinoxalin-2-yl)benzenesulfonamide derivative $6 \mathrm{a}$ was generally most 
active than the corresponding heterocycles, which presented $\mathrm{IC}_{50}=1.2 \mu \mathrm{M}$. It is notable that these compounds a new potential class of antimalarial agents.

As well as the overhead-defined antimalarial showing, toxicity studied was achieved on the samples that displayed an antimalarial activity less than $15 \mu \mathrm{M}$. Thus, $N$-(2-aminooxazol-

4-yl)benzenesulfonamide

$(3 \mathrm{c})$,

(4a),
$N$-(2H-benzo[b][1,4]oxazin-3-yl)-4-

$N$-(2H-benzo[b][1,4]thiazin-3-yl)-4-

chlorobenzenesulfonamide (5a), 4-chloro- $N$-(quinoxalin-2-yl)benzenesulfonamide (6a), 4methyl- $N$-(quinoxalin-2-yl)benzenesulfonamide

2-(pyridin-4-ylamino)- $N$ tosylacetamide (7b), $\mathrm{N}$-(phenylsulfonyl)-2-(pyridin-4-ylamino)acetamide $\quad$ (7c), 2-(1Hbenzo[ $d]$ imidazol-2-ylthio)- $N$-(4-chlorophenylsulfonyl)acetamide $\quad$ (9a) and $2-(1 H-$ benzo $[d]$ imidazol-2-ylthio)- $N$-(phenylsulfonyl)acetamide (9c) were subjected to further testing utilizing MTT-assay against [3-(4,5-dimethylthiazol-2-yl)-2,5-diphenyltetrazolium bromideassay]. The data of these compounds biological study are presented in Table 3.

Stimulatingly, the in vitro cytotoxicity results showed that only compounds $3 \mathrm{c}, 5 \mathrm{a}, 6 \mathrm{a}$, 7b, 9c, and 9a have SI's standards as displayed in Table 3, whereas all other compounds did not show cytotoxicity at the tested concentrations. In an instant of, the significance of substituted sulfonamide compounds as synthetic precursors for the biologically important class of drugs incorporating different heterocycles ring. The significance biology of these compounds was demonstrated via evaluation of their in vitro antiplasmodial activity and cytotoxicity, pointing to on talented potential of Sulfonamide derivatives as an innovative sort of antimalarial agent.

\subsubsection{Investigation of physicochemical properties.}

Advanced antimalarial selection with synthesized sulphonamide demonstrated dynamic in vitro against $p$. Falciparum with low cytotoxicity. Nevertheless, they required oral bioavailability due to their lowly ADMET profile. These detections motivated us to assess the physicochemical possessions from demanding our compounds, as shown in Table 4, which identified the exacerbates with perfect oral absorption properties with future structural modifications to move forward ADMET properties.

Table 3. The $\mathrm{IC}_{50}$ of in vitro for antiplasmodial activity and cytotoxicity.

\begin{tabular}{|c|c|c|c|}
\hline Compound & D 10: $\mathrm{IC}_{50}(\mu \mathrm{M})$ & CHO:IC ${ }_{50}(\mu \mathrm{M})$ & $\mathbf{S I}^{\mathbf{a}}$ \\
\hline $3 \mathbf{c}$ & 5.32 & 132.2 & 24.84 \\
\hline $4 \mathbf{a}$ & 11.25 & $\mathrm{NT}^{\mathrm{c}}$ & $\mathrm{ND}^{\mathrm{d}}$ \\
\hline $5 \mathbf{5 a}$ & 3.2 & 110.1 & 34.37 \\
\hline 6a & 1.2 & 98.3 & 81.91 \\
\hline $\mathbf{6 b}$ & 11.3 & NT & $\mathrm{ND}$ \\
\hline $7 \mathbf{b}$ & 9.41 & 145 & 15.4 \\
\hline $7 \mathrm{c}$ & 7.23 & 450.2 & 62.26 \\
\hline $9 a$ & 2.3 & 385.8 & 167.7 \\
\hline $9 c$ & 11.23 & NT & ND \\
\hline
\end{tabular}

a SI(Selectivity index); IC50 CHO/IC50 D10; number of data sets averaged $=\mathrm{Bn}$. cNT= non toxic at $100 \mu \mathrm{g} / \mathrm{ml}$. $\mathrm{dND}=$ not determined.

Table 4. Physicochemical characters of prepared sulfonamide compounds.

\begin{tabular}{|c|c|c|c|c|c|c|c|c|c|}
\hline Compound & $\begin{array}{c}\text { Molecular } \\
\text { Weight }\end{array}$ & pIC50 & $\begin{array}{c}\text { cLog } \\
\mathbf{P}\end{array}$ & $\mathbf{L i p E}^{\mathbf{a}}$ & Compound & $\begin{array}{c}\text { Molecular } \\
\text { Weight }\end{array}$ & $\mathrm{pIC}_{50}$ & $\begin{array}{c}\text { cLog } \\
\mathbf{P}\end{array}$ & $\operatorname{LipE}^{\mathbf{a}}$ \\
\hline $2 a$ & 289.75 & 4.47 & 1.60 & 2.87 & $6 \mathrm{~b}$ & 299.35 & 4.94 & 2.57 & 2.37 \\
\hline $2 \mathbf{b}$ & 269.34 & 4.61 & 1.20 & 3.41 & 6c & 285.32 & 4.56 & 2.11 & 2.45 \\
\hline $2 c$ & 255.31 & 4.35 & 0.74 & 3.61 & $7 \mathbf{a}$ & 325.77 & 4.09 & 0.83 & 3.26 \\
\hline $3 a$ & 273.69 & 4.33 & 1.04 & 3.29 & $7 b$ & 305.08 & 5.02 & 1.11 & 3.91 \\
\hline $3 b$ & 253.28 & 4.20 & 0.64 & 3.56 & $7 c$ & 291.33 & 5.14 & 0.65 & 4.49 \\
\hline $3 c$ & 239.25 & 5.27 & 0.18 & 5.09 & $\mathbf{8 a}$ & 398.89 & 4.34 & 3.29 & 1.05 \\
\hline \multicolumn{9}{|c|}{ https://biointerfaceresearch.com/ } & 1221 \\
\hline
\end{tabular}




\begin{tabular}{|c|c|c|c|c|c|c|c|c|c|}
\hline Compound & $\begin{array}{c}\text { Molecular } \\
\text { Weight }\end{array}$ & pIC50 & $\begin{array}{c}\text { cLog } \\
\text { P }\end{array}$ & $\operatorname{LipE}^{\mathbf{a}}$ & Compound & $\begin{array}{c}\text { Molecular } \\
\text { Weight }\end{array}$ & pIC50 & $\begin{array}{c}\text { cLog } \\
\text { P }\end{array}$ & LipE $^{a}$ \\
\hline $4 a$ & 322.76 & 4.94 & 2.76 & 2.18 & $8 b$ & 378.48 & 4.56 & 3.56 & 1.00 \\
\hline $4 b$ & 302.35 & 4.61 & 2.27 & 2.34 & $8 c$ & 364.45 & 4.47 & 3.10 & 1.37 \\
\hline $4 c$ & 288.32 & 4.77 & 1.81 & 2.96 & $9 a$ & 381.85 & 5.63 & 2.66 & 2.97 \\
\hline $5 \mathbf{a}$ & 338.82 & 5.49 & 3.33 & 2.16 & $9 \mathrm{~b}$ & 361.43 & 4.12 & 2.94 & 1.18 \\
\hline $5 \mathbf{b}$ & 318.41 & 4.51 & 2.85 & 1.66 & $9 \mathrm{c}$ & 347.41 & 4.94 & 2.48 & 2.46 \\
\hline $5 c$ & 304.38 & 4.25 & 2.38 & 1.87 & 6a & 319.76 & 5.92 & 2.96 & 2.96 \\
\hline $\operatorname{CQ}(n=8)^{b}$ & 515.86 & 7.76 & 3.73 & 4.03 & $\mathrm{CQ}(\mathrm{n}=8)^{b}$ & 5.15 .86 & 7.76 & 3.73 & 4.03 \\
\hline
\end{tabular}

LipE $=$ pIC $_{50}-\operatorname{cLogP} ;{ }^{b}$ chloroquine

There is an overall contract that the atomic characters for pill candidates, for instance, atomic, weight, and lipophilicity, essential for significant ADMET properties. Additional characterization, such as solubility, Freedom, and circulation volume, would be likewise dominant and reflect theon profiling of pill hopefuls. Also, the utilization of computational instruments requirements to be aided enhance medication regardless of a plan to accomplish a greater quantity.

To rationalize the drug discovery/development methodology Those Lipinski's 'Rule from claiming Five' and other parameters identical lipophilic. The efficiency of LipE brings been designated to make suitable devices to support over-picking oral drugs.

Synthesized heterocycles compounds had values of cLogP between 2 and 4 (mean value $\approx 2.23$ ) and molecular weights (MWs) below 400 (mean value $\approx 341.58$ ). [64] which recommended that compounds with a cLogP $<4$ and MWs below 400 have an additional promising ADMET outline. Figure 4 demonstrates the spreading of cLogP against Molecular weight for these compounds. Amongst them, five compounds ( $56 \%)$ had a cLogP between 2 and 4.

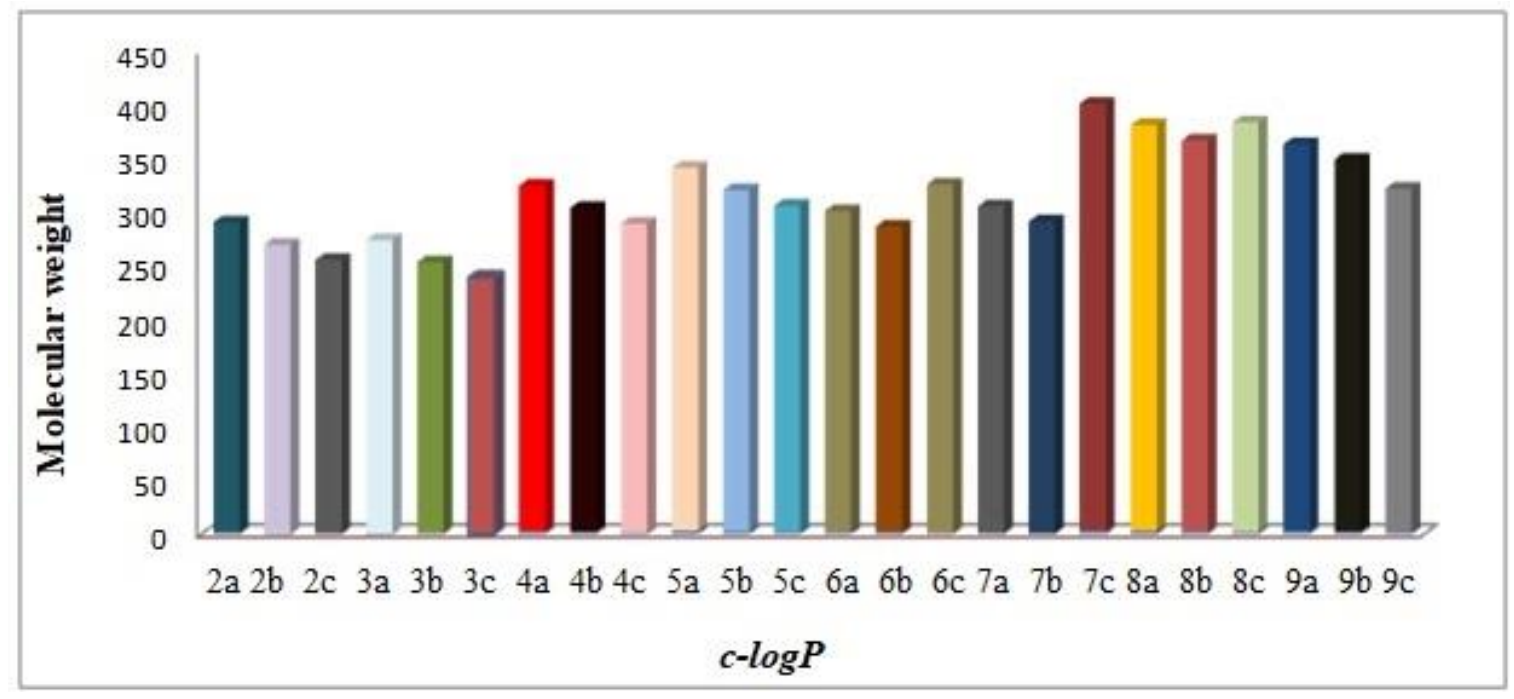

Figure 4. The cLogP against molecular weight $n$.

The relations between lipophilicity and MW which attractive in medication with higher in vitro control, whereas the high intensity needs specification for target with little drugs. Though the in vitro strength and lipophilicity of combinations were vital factors in evaluating the theory of Lipophilic Efficiency (LipE) assistances, we imagine the accustomed association between the potency in vitro and lipophilicity character of synthesized compounds [65] also the excellent lead mixes have higher LipE values. Plotting cLogP against $\mathrm{pIC}_{50}$ as displayed in Figure 5 The synthesized sulfonamide compounds showed that assumption along the lines of indistinguishable LipE numbers. Generally, the compounds have low LipE values, which have high cLogP values. 


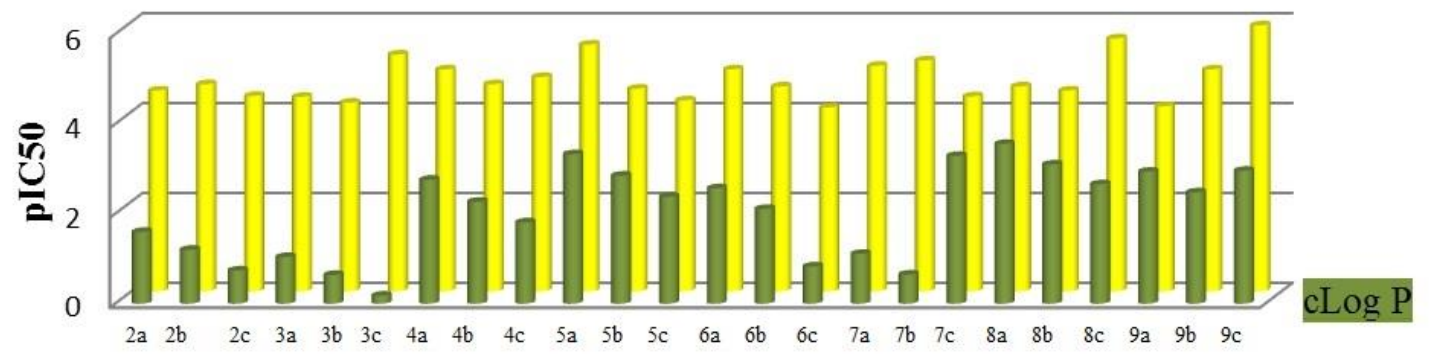

Figure 5. Plotting of $\mathrm{cLogP}$ againist $\mathrm{pIC}_{50}$ and LipE analysis.

Stimulating of observing that substitution of the quinoxaline group in 6a has similar potency of $\operatorname{LipE}$ and c-Log P values (2.96), but the c-LogP value of benzo[b][1,4]thiazin(5a) was (3.33) higher than LipE (2.16). Current animal care studies established that the hazard of decreased cLogP below 4. Furthermore, the suggestion of polar sides such as the nitrogen atom in quinoxaline moiety, which is important in binding site interactions of compound 6a. Definitely, $4 \mathrm{a}$ and 5a compounds had the almost maximum LipE worth of the data set and were thought that to be the most optimum heterocyclic. Also, docking outcomes indicated an extra binding interaction of $6 \mathrm{a}$ with the plasmepsins due to the occurrence of a quinoxaline moiety.

\subsection{Molecular docking study.}

3.3.1. Docking with plasmepsin-1and plasmepsin-2.

The Sulfonamide has been described to prevent plasmodia aspartate proteases and cysteine proteases; consequently, the performance docking to regulate the binding alignments of the synthesized sulphonamides $2 \mathrm{a}-9 \mathrm{c}$ at the energetic situates of amino acid excesses of the enzymes Plasmepsin-1 and plasmepsin-2. Moreover, the free bind energy of the examined compounds was assumed in Table 5. The synthesized sulphonamides were confirmed through molecular docking interactions with Plasmodium falciparum dihydrofolatereductasethymidylate synthase (PfDHFR-TS) wild-type complexes with WR99210, NADPH, and dUMP(1j3i) [42]. Plasmepsin-1 and Crystal structure of Pfg27 from Plasmodium falciparum(1n81), Plasmepsin-2 generated by AutoDockVina and observed via Discovery Studio program.

Table 5. Molecular docking of sulfonamide derivatives to Plasmepsin-1(1j3i)and Plasmepsin-2 (1n81).

\begin{tabular}{c|c|c|c|c|c} 
& \multicolumn{7}{|c}{$\mathbf{E}\left(\mathbf{K c a l}^{-m o l} \mathbf{l}^{-1}\right)$} \\
\hline Compound & Plasmepsin-1 & Plasmepsin-2 & $\mathbf{C o m p o u n d}$ & Plasmepsin-1 & Plasmepsin-2 \\
\hline $\mathbf{2 a}$ & -5.69 & -6.53 & $\mathbf{6 a}$ & -4.6 & -6.2 \\
\hline $\mathbf{2 b}$ & 14.35 & -6.7 & $\mathbf{6 b}$ & 19.5 & -6.76 \\
\hline $\mathbf{2 c}$ & 11.95 & -6.46 & $\mathbf{6 c}$ & 6.91 & -6.83 \\
\hline $\mathbf{3 a}$ & -5.39 & -5.8 & $\mathbf{7 a}$ & 24.68 & -4.97 \\
\hline $\mathbf{3 b}$ & 2.74 & -5.64 & $\mathbf{7 b}$ & 26.5 & -3.85 \\
\hline $\mathbf{3 c}$ & 273.62 & -5.43 & $\mathbf{7 c}$ & 24.16 & -5.81 \\
\hline $\mathbf{4 a}$ & -5.63 & -6.49 & $\mathbf{8 a}$ & 334.42 & 450.32 \\
\hline $\mathbf{4 b}$ & 45.06 & -5.67 & $\mathbf{8 b}$ & 249.0 & -7.23 \\
\hline $\mathbf{4 c}$ & 41.37 & -6.04 & $\mathbf{8 c}$ & 145.43 & -6.85 \\
\hline $\mathbf{5 a}$ & -5.3 & -6.10 & $\mathbf{9 a}$ & 137.07 & -5.8 \\
\hline $\mathbf{5 b}$ & 52.43 & -7.23 & $\mathbf{9 b}$ & 118.06 & -6.96 \\
\hline $\mathbf{5 c}$ & -3.4 & -6.6 & $\mathbf{9 c}$ & 87.6 & -6.86
\end{tabular}


Free bind energy of compound $5 \mathrm{c}$, determined to be the greatest optimum benzenesulfonamide derivative, for Plasmepsin-2(-7.6 Kcal $/ \mathrm{mol})$, while compound 6a with plasmepsin-2 showed $(-6.12 \mathrm{Kcal} / \mathrm{mol})$ and plasmepsin-1 $(-4.6 \mathrm{Kcal} / \mathrm{mol})$. Compound $4 \mathrm{a}$ showed the most binding energy of both Plasmepsin-1 $(--5.63 \mathrm{Kcal} / \mathrm{mol})$ with highlights a short distance $(3.4 \AA)(3.6 \AA)$ and two hydrogen atom linked with 1j3i:Tyr64:O and 1j3i:Lys 160:HZ3 and for Plasmepsin-2 (-6.49Kcal/mol) with short distance $(2.6 \AA)$ and one hydrogen atom linked with 1N81:A:GLN130:OE1 showed a better interaction between the carbonyl of sulfonamide and GLN 130 as shown in Figure 6.
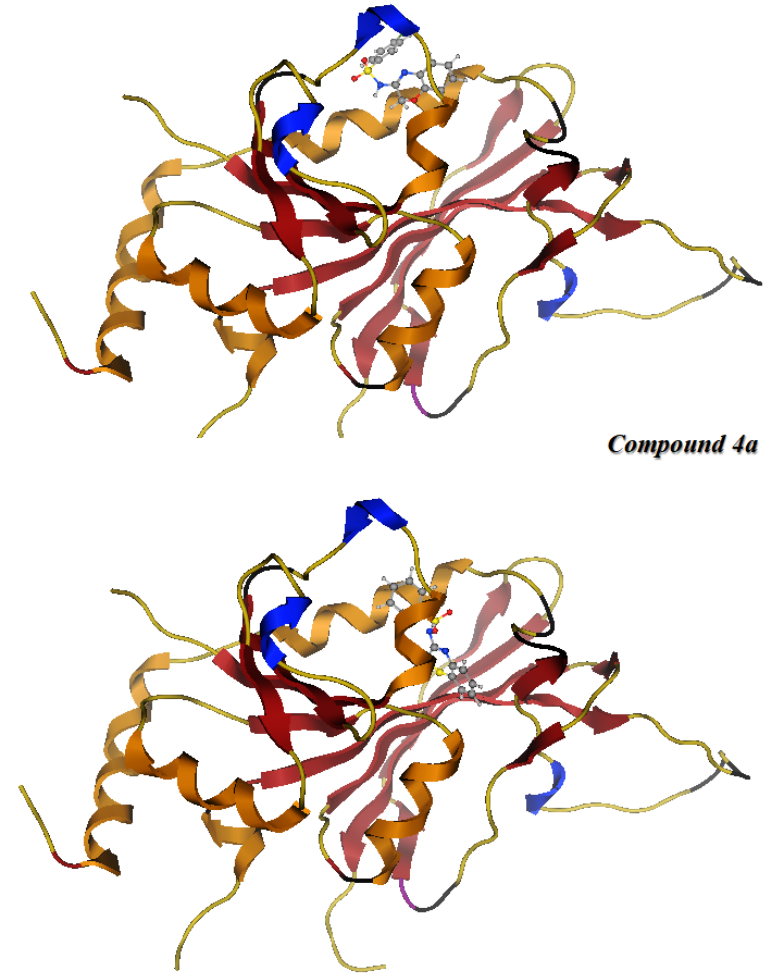

Compound 5c

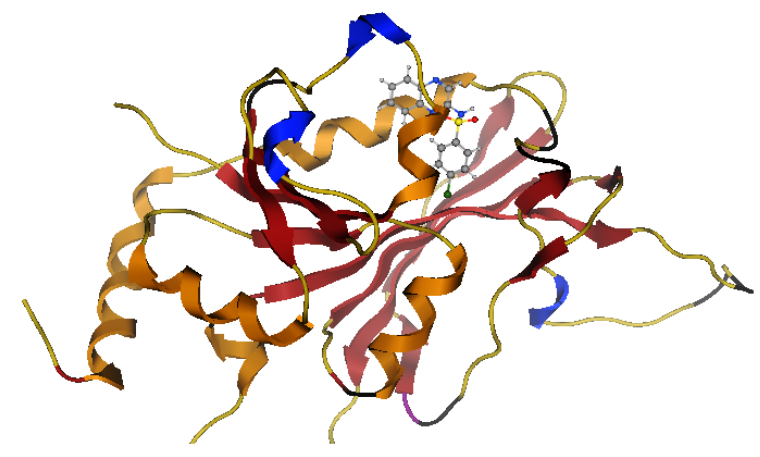

Compound 6a

(a)

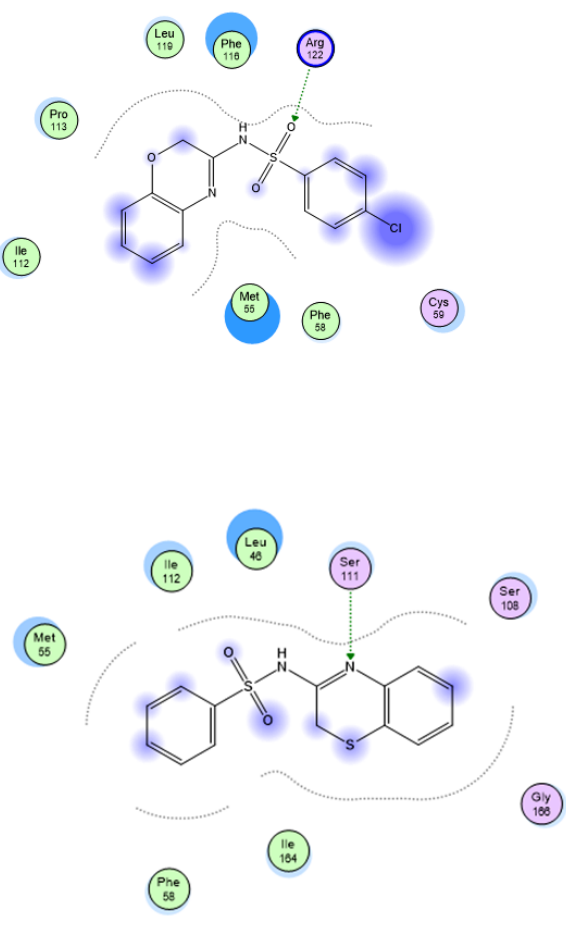

(iii)

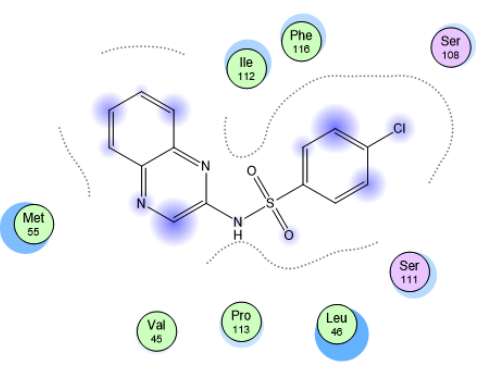




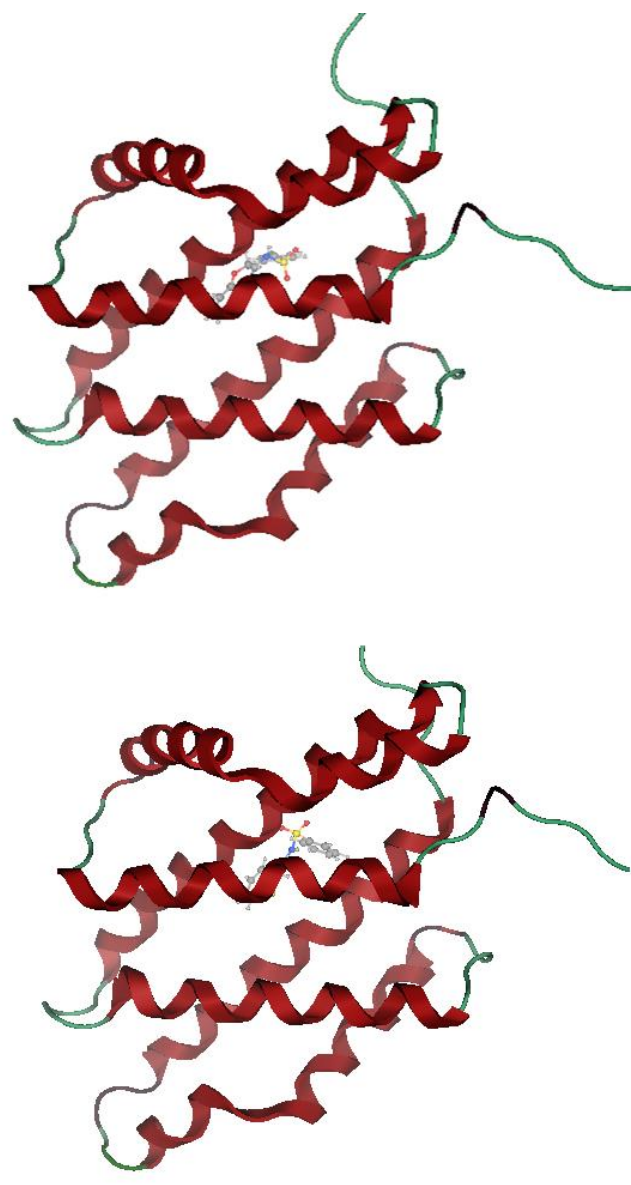

$(120)$<smiles>[C+]=[C-]</smiles>

(s:)

(39)<smiles>[C-]1CC1</smiles>

Compound $4 a$

\section{; :}

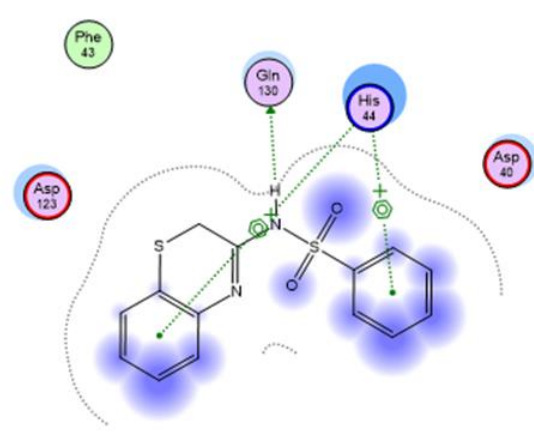

Compound $5 \mathrm{c}$
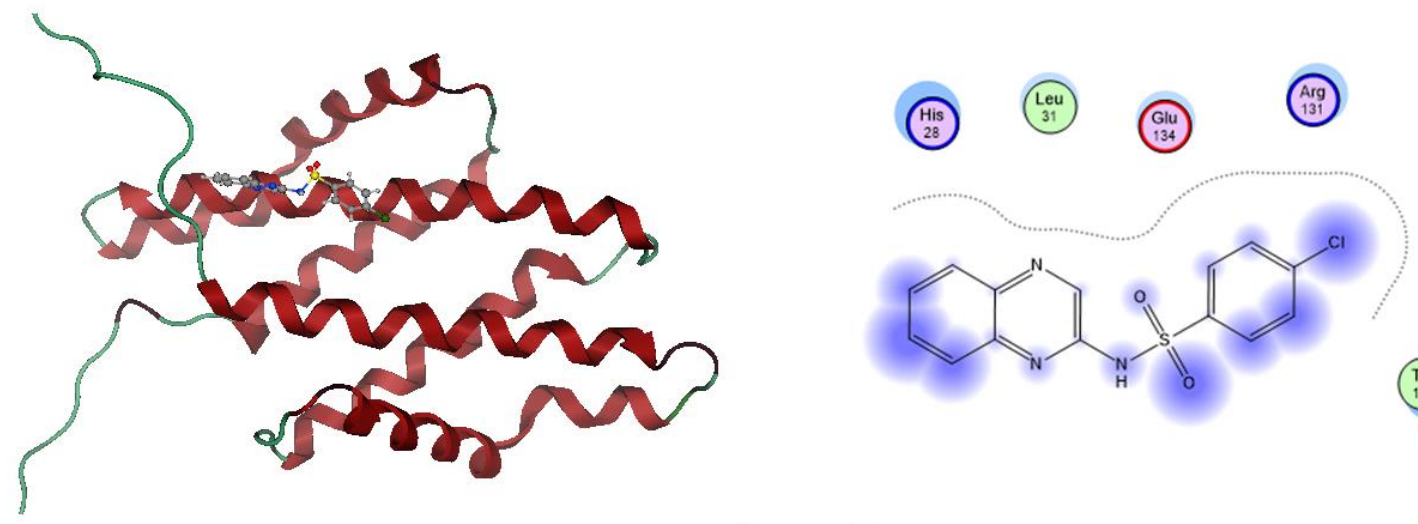

Compound $6 a$

(b)

Figure 6. (a) Docking of compound4a, 5c and 6awith Plasmepsin-1(PDBID:13ji); (b) Molecular docking of 4a, 5c and 6awith Plasmepsin-2(PDBID:1n81).

Furthermore, the Compound 5c docked into Plasmepsin-2 (1n81) binding site with binding energy (-6.6) and total energy internal (-8.03) and torsion energy (0.6) and highlights a short distance $(2.6 \AA)$ 1n81:Gln130:OE1, 7C Lig1:N1 where the $\mathrm{NH}$ of $\mathrm{N}-(2 \mathrm{H}$ benzo[b][1,4]thiazin-3-yl)benzenesulfonamide (5c) as a Michael acceptor with protein residue Gln130 at the binding site.

Moreover, the Compound 6a docked into the (1j31)Plasmepsin-1 with binding energy (45.55), and total energy internal (5.71) and torsion energy(0.89) and places of interest a short bond length $(2.7 \AA)$ Highlighting the $H$-bond of quinoxaline moiety with a residue of proteins. Also, the extra bind energy interaction of compound 6a with plasmepsin-2 is displayed in Figure 6. Besides, molecular docking outcomes recommend a Michael reaction mechanism 
between these ligands and proteases binding energy (-6.12) and total energy internal (-.64) and torsion energy (0.89)and highpoints a short length $(2.1 \AA)$.

\subsubsection{Docking of sulfonamide derivatives with SARS-CoV-2.}

Molecular Docking model studies were also done to calculate the binding mode of drugs 4a, 5c, and 6a at the SARS-CoV-2 Mpropocket utilizing (Crystal structure of COVID19 complex with inhibitor $\mathrm{N}_{3}$ (PDBID:6lu7) and Closed Structure of SARS-CoV-2 spike glycoprotein, Function, and Antigenicity of the SARS-CoV-2 Spike Glycoprotein.(PDBID:6vxx) as displayed in Table 6.

The binding affinity of compounds $4 \mathrm{a}, 5 \mathrm{c}$, and $6 \mathrm{a}$ with (PDBID:6lu7) were ranging from -6.0 to $-5.5 \mathrm{kcal} / \mathrm{mol}$ and with bond distance ranging from 1.03-1.97 $\AA$ where the compound 4-Chloro-N-(quinoxalin-2-yl)benzenesulfonamide(6a) showed the less binding affinity to the pocket of the complex of SARS-Cov-2 with Asp 289, Glu 288, Thr 199, leu 272, Tyr237as shown in Figure 7.

Table 6. The biological docking of compounds 4a, 5c, and 6a to (PDBID:6lu7) and (PDBID:6vxx)

\begin{tabular}{|c|c|c|c|c|c|c|c|}
\hline \multicolumn{5}{|c|}{ (PDB:6lu7) } & \multicolumn{3}{|c|}{$(P D B: 6 v x x)$} \\
\hline Compound & $\begin{array}{c}\text { Enerey } \\
\text { affinity(kcal/mol) }\end{array}$ & Distance $(\AA)$ & Amino acids & & $\begin{array}{c}\text { Energy affinity } \\
\text { (kcal/mol) }\end{array}$ & Distance $(\AA)$ & Amino acids \\
\hline $4 \mathbf{a}$ & $(-6.0 \quad \mathrm{kcal} / \mathrm{mol})$ & $1.526 \AA$ & $\begin{array}{l}\text { Thr 199, Leu 286, } \\
\text { Leu 287, Arg 131, } \\
\text { Glu 288, Glu 290, } \\
\text { Asp 289 }\end{array}$ & $4 a$ & $(-4,788 \quad \mathrm{kcal} / \mathrm{mol})$ & $1.819 \AA$ & $\begin{array}{l}\text { Arg 357, Ala 958, } \\
\text { Pro 579, Pro330, } \\
\text { Pro 521 }\end{array}$ \\
\hline $5 c$ & $(-5.5 \mathrm{kcal} / \mathrm{mol})$ & $1.978 \AA$ & $\begin{array}{l}\text { Leu } 287, \text { Thr } 199, \\
\text { Leu 286, Tyr 239, } \\
\text { Arg } 131, \text { Lys } 137, \\
\text { Asp } 289, \text { Gln } 290\end{array}$ & $5 c$ & $(-8.11 \mathrm{kcal} / \mathrm{mol})$ & $1.420 \AA$ & $\begin{array}{l}\text { Arg 1014, Gln } \\
\text { 957, Thr 961, Ser } \\
\text { 1003, Thr 1006, } \\
\text { Gln 965 }\end{array}$ \\
\hline $\mathbf{6 a}$ & $(-6.0 \mathrm{kcal} / \mathrm{mol})$ & $1.038 \AA \dot{~}$ & $\begin{array}{l}\text { Asp 289, Glu 288, } \\
\text { Thr 199, leu 272, } \\
\text { Tyr237 }\end{array}$ & 6a & $(-5.94 \mathrm{kcal} / \mathrm{mol})$ & $1.956 \AA$ & $\begin{array}{l}\text { Asp 574, Arg 567, } \\
\text { Gly 566, Asp 568, } \\
\text { Lys } 557\end{array}$ \\
\hline
\end{tabular}
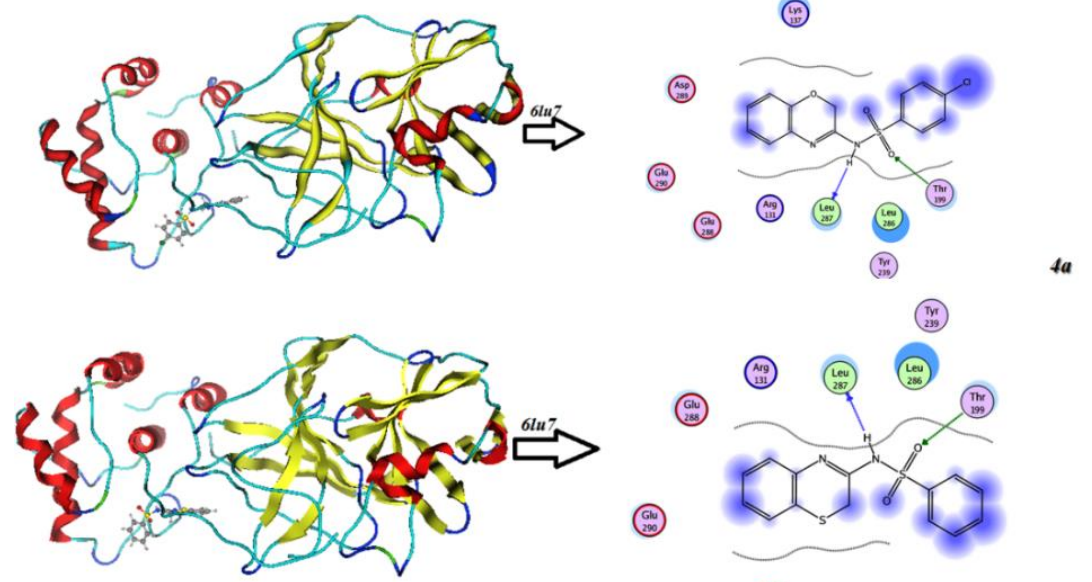

(ax)
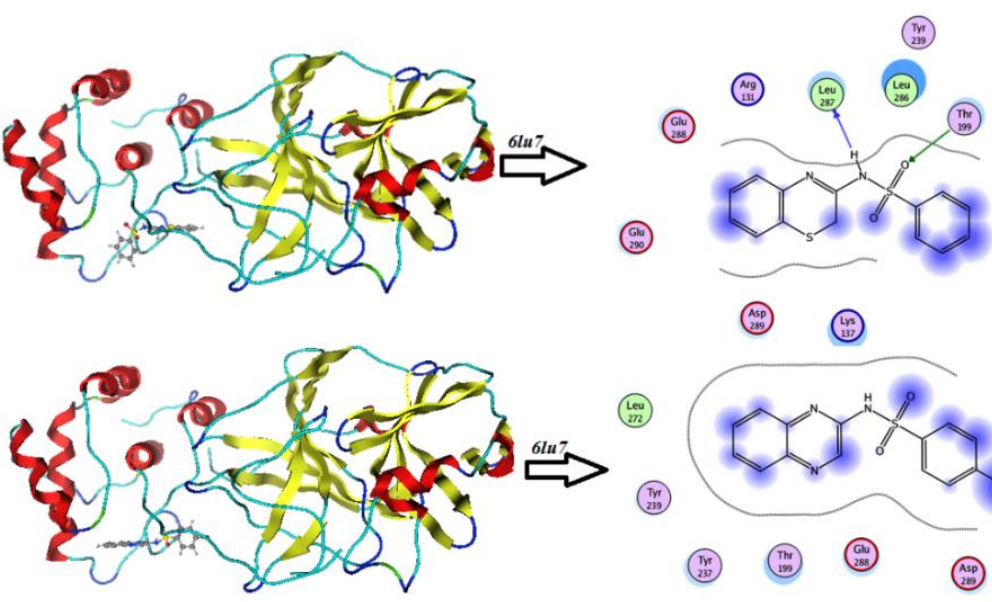

(장) (궁

Lew

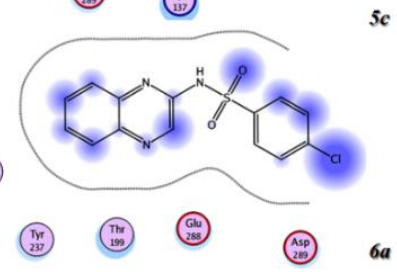


(Pro

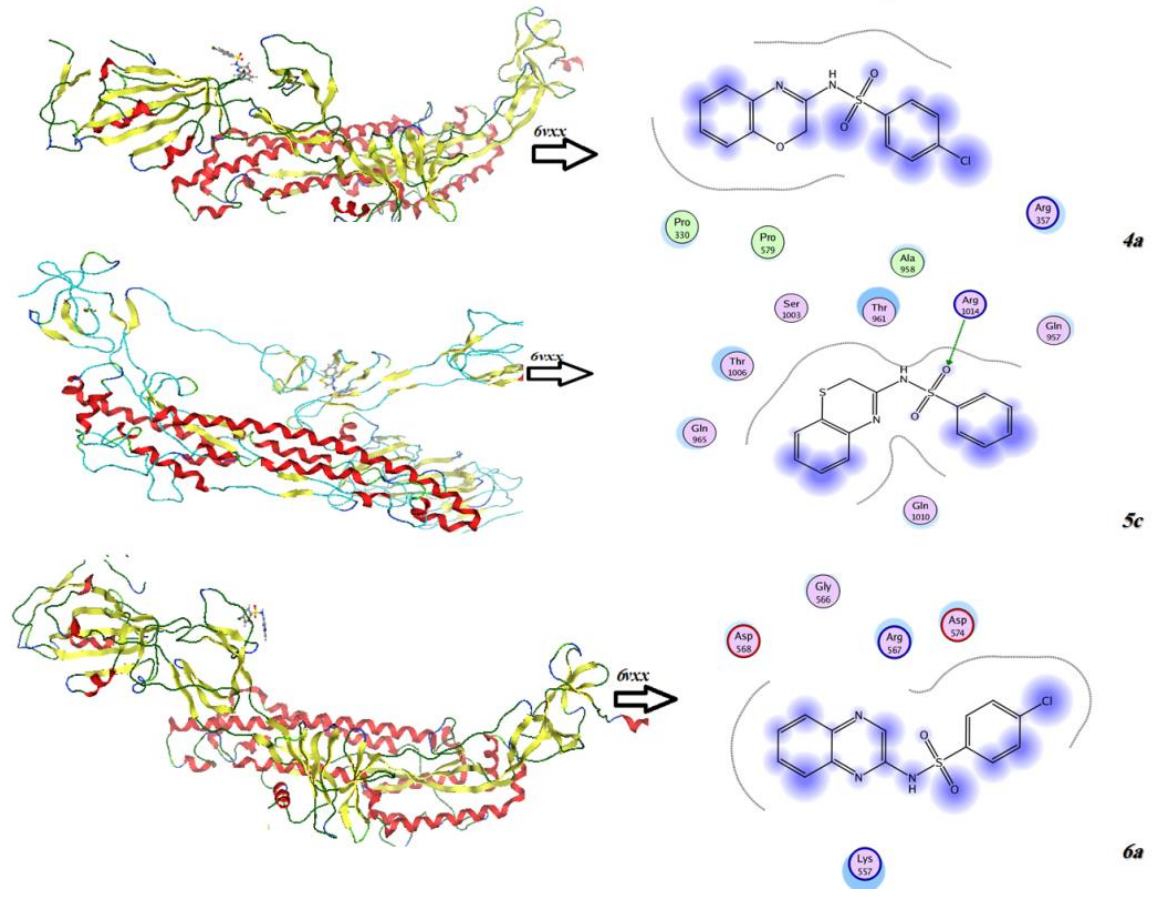

Figure 7. Binding models of 4a, 5c and 6a with (a)(PDBID:6lu7),(b)(PDIDB:6vxx).

Furthermore, the docking of compounds 4a, 5c, and 6a with Closed state Structure of the SARS-CoV-2 spike glycoprotein, Function, and Antigenicity of the SARS-CoV-2 Spike Glycoprotein(PDBID:6vxx) displayed the mandatory energy from $-8.11 \mathrm{kcal} / \mathrm{mol}$ to -4.7 $\mathrm{kcal} / \mathrm{mol}$ and with bond distance ranging from 1.42-1.95 $\AA$ where compound $5 \mathrm{c}$ showed less

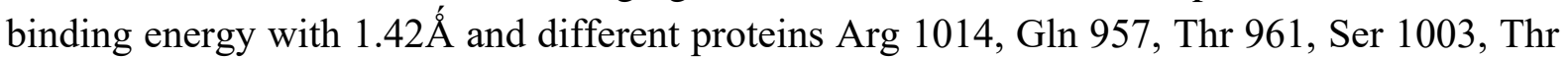
1006, Gln 965, the compound 6a has the shortest bond distance with 1.956 ̊, with Asp 574, Arg 567, Gly 566, Asp 568, Lys 557as displayed in Table 6 and Figure 7.

\section{Conclusions}

In conclusion of our work, the activities of novel fused sulfonamide derivatives anticipated antimalarial activity. The SAR relationship related to the most active compound was different fused heterocycles with sulfonamide with $\mathrm{IC}_{50}=11.25,3.2,5.23$, and $1.2 \mu \mathrm{g} / \mathrm{ml}$ for compounds $4 \mathrm{a}, 5 \mathrm{a}, 5 \mathrm{c}$, and $6 \mathrm{a}$, respectively. Furthermore, their cLogP values between 2 and 4 and the attendance of polar nitrogen groups in quinoxaline ring find the bind site interactions of compound 6a. Moreover, the optimized molecular structure of compounds $4 \mathrm{a}, 5 \mathrm{c}, 6 \mathrm{a}$ utilizing DFT/B3LYP/6-31G(d) function supports their stability, and the geometric characters confirm their reactivity. Furthermore, the docking studies of these compounds against Plasmepsin-1, Plasmepsin-2, and SARS-CoV-2 showed different potential activities and short hydrogen bond distances.

\section{Funding}

This research received no external funding.

\section{Acknowledgments}

The authors thanks to the Green chemistry department in N R C Egypt and the Ministry of Health for biological activities 


\section{Conflicts of Interest}

The authors declare no conflict of interest.

\section{References}

1. Aboelnaga, A.; Fahim, A.M.; El-Sayed, T.H. Computer Aid Screening for Potential Antimalarial Choroquinone Compounds as Covid 19 Utilizing Computational Calculations and Molecular Docking Study. OnLine Journal of Biological Sciences 2020, 20, 207-220, https://doi.org/10.3844/ojbsci.2020.207.220.

2. Akl, E.M.; Dacrory, S.; Abdel-Aziz, M.S.; Kamel, S.; Fahim, A.M. Preparation and characterization of novel antibacterial blended films based on modified carboxymethyl cellulose/phenolic compounds. Polymer Bulletin 2020, 78, 1061-1085, https://doi.org/10.1007/s00289-020-03148-w.

3. Alsulaili, A.D.; Fahim, A.M. Oil removal from produced water by agriculture waste adsorbents. International Journal of Environment and Waste Management 2020, 25, https://doi.org/10.1504/ijewm.2020.104345.

4. Dacrory, S.; Fahim, A.M. Synthesis, anti-proliferative activity, computational studies of tetrazole cellulose utilizing different homogenous catalyst. Carbohydr Polym 2020, https://doi.org/10.1016/j.carbpol.2019.115537.

5. Fahim, A.; Ismael, E. Synthesis, antimicrobial activity and quantum calculations of Novel sulphonamide derivatives. Egyptian Journal of Chemistry 2019, 7, 1427-1440, https://doi.org/10.21608/ejchem.2019.6870.1575.

6. Fahim, A.M.; Farag, A.M. Synthesis, antimicrobial evaluation, molecular docking and theoretical calculations of novel pyrazolo[1,5-a]pyrimidine derivatives. Journal of Molecular Structure 2020, 1199, https://doi.org/10.1016/j.molstruc.2019.127025.

7. Fahim, A.M.; Farag, A.M.; Mermer, A.; Bayrak, H.; Şirin, Y. Synthesis of Novel $\beta$-lactams: Antioxidant activity, Acetylcholinesterase Inhibition and Computational Studies. Journal of Molecular Structure 2021, 1233, https://doi.org/10.1016/j.molstruc.2021.130092.

8. Fahim, A.; Farag, A.M.; Shaaban, M.; Ragab, E. "Synthesis and DFT study of novel pyrazole,thiophene, 1,3-thiazole and 1,3,4-thiadiazole derivatives". European Journal of Chemistry 2018, 1, https://doi.org/10.5155/eurjchem.9.1.30-38.1675.

9. Fahim, A.M.; Shalaby, M.A. Synthesis, biological evaluation, molecular docking and DFT calculations of novel benzenesulfonamide derivatives. Journal of Molecular Structure 2019, 1176, 408-421, https://doi.org/10.1016/j.molstruc.2018.08.087.

10. Fahim, A.M.; Shalaby, M.A.; Ibrahim, M.A. Microwave-assisted synthesis of novel 5-aminouracil-based compound with DFT calculations. Journal of Molecular Structure 2019, 1194, 211-226, https://doi.org/10.1016/j.molstruc.2019.04.078.

11. Fahim, A.M.; Wasiniak, B.; Łukaszewicz, J.P. Molecularly Imprinted Polymer and Computational Study of (E)-4-(2- cyano-3-(dimethylamino)acryloyl)benzoic Acid from Poly(ethylene terephthalate) Plastic Waste. Current Analytical Chemistry 2020, 16, 119-137, https://doi.org/10.2174/1573411015666190131123843.

12. Farag, A.M.; Fahim, A.M. Synthesis, biological evaluation and DFT calculation of novel pyrazole and pyrimidine derivatives. Journal of Molecular Structure 2019, 1179, 304-314, https://doi.org/10.1016/j.molstruc.2018.11.008.

13. Jin, Z.; Du, X.; Xu, Y.; Deng, Y.; Liu, M.; Zhao, Y.; Zhang, B.; Li, X.; Zhang, L.; Peng, C.; Duan, Y.; Yu, J.; Wang, L.; Yang, K.; Liu, F.; Jiang, R.; Yang, X.; You, T.; Liu, X.; Yang, X.; Bai, F.; Liu, H.; Liu, X.; Guddat, L.W.; Xu, W.; Xiao, G.; Qin, C.; Shi, Z.; Jiang, H.; Rao, Z.; Yang, H. Structure of Mpro from SARS-CoV-2 and discovery of its inhibitors. Nature 2020, 582, 289-293, https://doi.org/10.1038/s41586020-2223-y.

14. Jo, S.; Kim, S.; Shin, D.H.; Kim, M.S. Inhibition of SARS-CoV 3CL protease by flavonoids. Journal of enzyme inhibition and medicinal chemistry $\mathbf{2 0 2 0}$ 35, 145-151, https://doi.org/10.1080/14756366.2019.1690480.

15. Kaya, S.; Gökce, H.; El-Azab, A.S.; Sert, Y.; Alanazi, M.M.; Öztürk, N.; Al-Agamy, M.H.M.; Abdel-Aziz, A.A.-M. Structural, Spectroscopic, Electronic and Molecular Docking Studies on (11R,12 S)-16Aminotetracyclo[6.6.2.02,7.09,14]hexadeca-2(7),3,5,9(14),10,12-hexaen-15-ol. ChemistrySelect 2019, 4, 825-837, https://doi.org/10.1002/slct.201803732.

16. Legros, J.-Y.; Toffano, M.; Fiaud, J.-C. Palladium-catalyzed substitution of esters of naphthylmethanols, 1naphthylethanols, and analogues by sodium dimethyl malonate. Stereoselective synthesis from enantiomerically pure substrates. Tetrahedron 1995, 51, 3235-3246, https://doi.org/10.1016/00404020(95)00061-C.

17. Li, H.-Y.; Wang, Y.; Yan, L.; Campbell, R.M.; Anderson, B.D.; Wagner, J.R.; Yingling, J.M. Novel and potent transforming growth factor beta type I receptor kinase domain inhibitor: 7-amino 4-(2-pyridin-2-yl5,6-dihydro-4H-pyrrolo[1,2-b]pyrazol-3-yl)-quinolines. Bioorg Med Chem Lett 2004, 14, 3585-3588, https://doi.org/10.1016/j.bmcl.2004.04.065. 
18. Masibi, K.K.; Fayemi, O.E.; Adekunle, A.S.; Al-Mohaimeed, A.M.; Fahim, A.M.; Mamba, B.B.; Ebenso, E.E. Electrochemical Detection of Endosulfan Using an AONP-PANI-SWCNT Modified Glassy Carbon Electrode. Materials 2021, 14, https://doi.org/10.3390/ma14040723.

19. Masood, S.; Rehman, W.; Khan, Z.; Arshad, H.; Begum, S.; Perveen, A. Structure Breaking/Making Property of Acefylline Piperazine in Aqueous, Aqueous Methanol, and Aqueous Ethylene Glycol Systems. Journal of Structural Chemistry 2018, 59, 1148-1157, https://doi.org/10.1134/S0022476618050189.

20. Mohamed, A.; Fahim, A.M.; Ibrahim, M.A. Theoretical investigation on hydrogen bond interaction between adrenaline and hydrogen sulfide. Journal of Molecular Modeling 2020, 26, https://doi.org/10.1007/s00894020-04602-2.

21. Mondal, S.; Mandal, S.M.; Mondal, T.K.; Sinha, C. Spectroscopic characterization, antimicrobial activity, DFT computation and docking studies of sulfonamide Schiff bases. Journal of Molecular Structure 2017, 1127, 557-567, https://doi.org/10.1016/j.molstruc.2016.08.011.

22. Morris, G.M.; Huey, R.; Lindstrom, W.; Sanner, M.F.; Belew, R.K.; Goodsell, D.S.; Olson, A.J. AutoDock4 and AutoDockTools4: Automated docking with selective receptor flexibility. Journal of Computational Chemistry 2009, 30, 2785-2791, https://doi.org/10.1002/jcc.21256.

23. Nshakira, N.; Kristensen, M.; Ssali, F.; Whyte, S.R. Appropriate treatment of malaria? Use of antimalarial drugs for children's fevers in district medical units, drug shops and homes in eastern Uganda. Tropical medicine \& international health: TM \& IH 2002, 7, 309-316, https://doi.org/10.1046/j.13653156.2002.00858.x.

24. Fahim, A.; Farag, A.M.; Nawwar, G.; Yakout, E.S.; Ragab, E. Synthesis and DFT calculations of azaMichael adducts obtained from degradation poly(methyl methacrylate) plastic wastes. International Journal of Environment and Waste Management 2019, 24, 337-353, https://doi.org/10.1504/IJEWM.2019.103641.

25. Soror, S.; Fahim, A.M.; Elabbady, S.; Nassar, E.; Aboelnaga, A. Synthesis, antimicrobial activities, docking studies and computational calculations of new bis-1,4-phenylene -1H-1,2,3-triazole derivatives utilized ultrasonic energy. Journal of Biomolecular Structure and Dynamics 2021, 1-18, https://doi.org/10.1080/07391102.2021.1875051.

26. Tadigoppula, N.; Korthikunta, V.; Gupta, S.; Kancharla, P.; Khaliq, T.; Soni, A.; Srivastava, R.K.; Srivastava, K.; Puri, S.K.; Raju, K.S.R.; Wahajuddin; Sijwali, P.S.; Kumar, V.; Mohammad, I.S. Synthesis and Insight into the Structure-Activity Relationships of Chalcones as Antimalarial Agents. Journal of Medicinal Chemistry 2013, 56, 31-45, https://doi.org/10.1021/jm300588j.

27. Zayed, E.M.; Zayed, M.A.; Fahim, A.M.; El-Samahy, F.A. Synthesis of novel macrocyclic Schiff's-base and its complexes having N2O2 group of donor atoms. Characterization and anticancer screening are studied. Applied Organometallic Chemistry 2017, 31, https://doi.org/https://doi.org/10.1002/aoc.3694.

28. Zhao, S.; Lin, Q.; Ran, J.; Musa, S.S.; Yang, G.; Wang, W.; Lou, Y.; Gao, D.; Yang, L.; He, D.; Wang, M.H. Preliminary estimation of the basic reproduction number of novel coronavirus (2019-nCoV) in China, from 2019 to 2020: A data-driven analysis in the early phase of the outbreak. International Journal of Infectious Diseases 2020, 92, 214-217, https://doi.org/10.1016/j.ijid.2020.01.050. 